\title{
Non-perturbative field theory: Progress in lattice field theory
}

\author{
P. Hernández \\ Universidad de Valencia and IFIC, Edificio de Institutos de Paterna, 46100 Burjassot, Valencia
}

Received: 14 January 2004 / Accepted: 8 February 2004 /

Published Online: 25 February 2004 - (C) Springer-Verlag / Società Italiana di Fisica 2004

\begin{abstract}
A very significant progress has been achieved in lattice field theory in recent years. This has been possible thanks to the spectacular increase in the computer speed, but most importantly by the development of new algorithms, the use of improved actions and the ingenuity of the questions being asked. I will illustrate the recent progress in the field by considering a few selected examples of these.
\end{abstract}

PACS. PACS-11.15.Ha Lattice gauge theory - PACS-12.38.Gc Lattice QCD calculations

\section{Introduction}

Lattice Field Theory is a method to study field theories from first principles and beyond perturbation theory. A prototype field theory that needs to be treated by nonperturbative means is QCD, where from the very simple Lagrangian, which only depends on the gauge coupling and the quark masses:

$$
\mathcal{L}_{Q C D}=-\frac{1}{2 g_{0}^{2}} \operatorname{Tr}\left\{F_{\mu \nu} F_{\mu \nu}\right\}+\sum_{f} \bar{\Psi}_{f}\left\{D+m_{f}\right\} \Psi_{f},
$$

one can in principle obtain a large number of predictions.

The starting point is the discretization of this Lagrangian in a space-time lattice in a way that preserves gauge invariance, locality and unitarity. The chiral symmetry of the QCD Lagrangian is unfortunately hard to implement, because of the famous Nielsen-Ninomiya theorem [1]. The most commonly used regularizations overcome this problem by having an explicit breaking of the symmetry (Wilson fermions) or by keeping a $U(1)$ subgroup at the price of breaking the flavour group completely (staggered fermions).

In either case the computation of observables via the path integral formulation becomes a finite calculation:

$$
\langle\mathcal{O}\rangle=\frac{\int D\left[A_{\mu}\right] \mathcal{O} \operatorname{det}\left(D+m_{f}\right) e^{-S_{\text {gauge }}}}{\int D\left[A_{\mu}\right] \operatorname{det}\left(D+m_{f}\right) e^{-S_{\text {gauge }}}} .
$$

However this calculation in $S U(3)$ involves $(T / a) \times(L / a)^{3}$ $\times 8 \times 4$ integrals, which for a reasonable lattice spacing $a=0.1 \mathrm{fm}$ (or inverse cutoff) and volume $L=2 \mathrm{fm}(L / a=$ $20)$ is an impossible task. Statistical methods such as the Montecarlo approach are necessary.

Universality warranties that, once we properly renormalize and take the continuum limit, $a \rightarrow 0$, we obtain results in QCD that are exact up to statistical errors, provided the chiral symmetry is properly restored. In the case of Wilson fermions, where the breaking of the symmetry is explicit, it is necessary to fine tune the couplings to ensure that Ward identities are restored in the continuum limit. This is by now a well understood procedure. In the case of staggered fermions, the naive continuum limit is chiral, but the number of flavours is naturally a multiple of $N_{f}=4$. Getting rid of the additional flavours requires modifications that do not obviously preserve locality:

$$
\operatorname{det}\left(D+m_{f}\right) \rightarrow\left[\operatorname{det}\left(D_{\text {staggered }}+m_{f}\right)\right]^{N_{f} / 4} .
$$

In spite of the remarkable progress in lattice QCD of the recent years, quenching remains an embarrassing but necessary approximation in many situations. This approximation amounts to neglecting fermion loops in the path integral, i.e. making the substitution

$$
\operatorname{det}\left(D+m_{f}\right) \rightarrow 1
$$

in (21). Although this approximation introduces a systematic error that cannot be estimated, experience shows that results are not far from experiment and it is thus a very good testing ground for the methods and techniques.

Progress in this field would not have been possible without the exponential growth of the computer speed, which is illustrated in Fig. 1 This progress has permitted for instance that quenched QCD simulations can now be performed in small PC clusters affordable to smaller research groups.

However, the increase in computer power has not been enough. Other aspects have been equally essential:

- Numerical algorithms which can get you orders of magnitude as illustrated in Fig. 1

- Improved actions: all lattice actions that preserve the symmetries of the continuum theory and have the same particle content lead to the same continuum limit, however you can speedup the approach to this limit by choosing a better action 


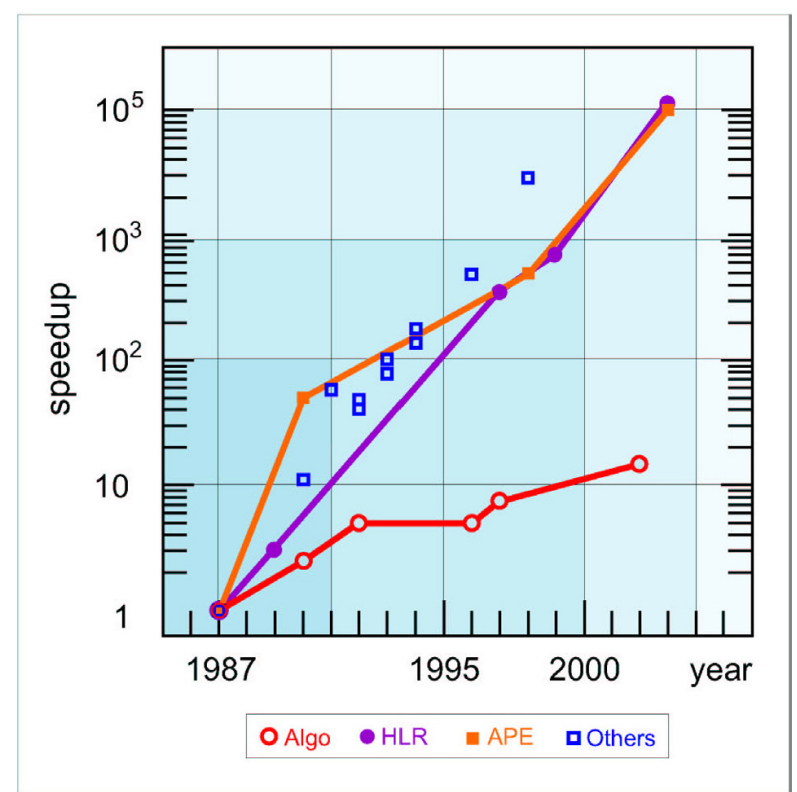

Fig. 1. Increase in computational speed for different platforms as a function of the year in the last ten years and normalized to the situation of 1987, compared to the gain due to algorithmic development (open circles). Taken from [2]

- Asking the right questions: the lattice allows us to do experiments that we cannot do in the laboratory. We can setup the degrees of freedom in QCD in different (unphysical) conditions that are useful to prove different properties of the system. We can for instance consider a finite volume, quark masses that differ from the physical ones, fixed topological sectors.

I will illustrate recent progress in the field by showing some selected examples of all these three points.

Unfortunately I cannot cover in this review all the interesting results in this field $(\mathcal{O}(250)$ contributions to Lattice2003 3]). I will mostly concentrate in QCD. In particular I will not cover the promising new ideas to deal with SUSY theories on the lattice 4]. Neither I will provide new world averages for the quantities relevant in CKM fits. For these and other interesting topics see the contributions to this conference $[5,6,7,8,8,9$.

\section{Algorithmic improvements}

\subsection{Confinement and the QCD string}

It is an old idea that non-abelian Yang-Mills theories could be (effective) string theories. This could explain confinement or the linear growth of the potential with the distance between an infinitely heavy (static) quark and antiquark

$$
\lim _{r \rightarrow \infty} V(r)=\sigma r \quad \sigma: \text { string tension, }
$$

as well as the fact that hadronic resonances fall approximately in Regge trajectories, satisfying a proportionality

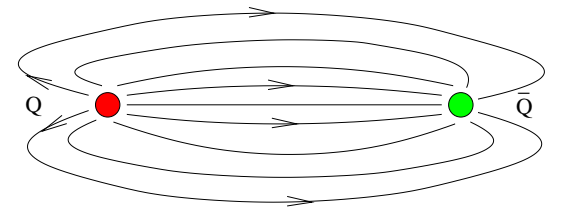

Fig. 2. Flux tube between static color charges

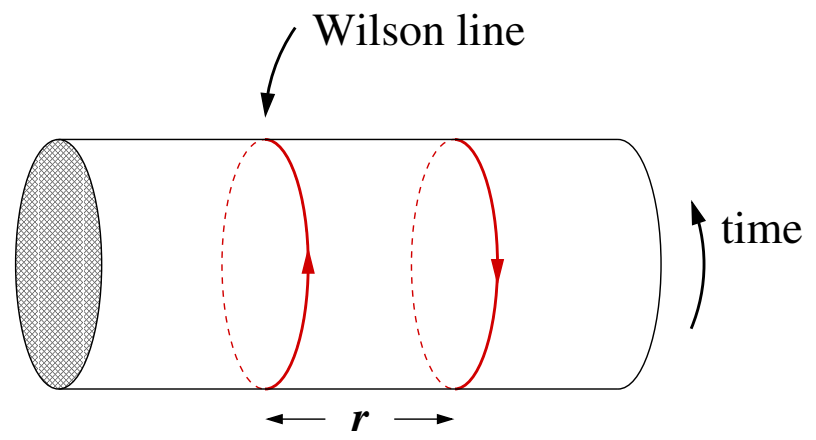

Fig. 3. Correlator of Polyakov loops

between their total angular momentum and their mass squared:

$$
J_{i} \sim m_{i}^{2}
$$

In its less ambitious formulation, this correspondence implies that the low energy degrees of freedom are the "stringy" excitations of the thin flux tube that forms linking color charges (see Fig. 2). One can then write the most general action for these degrees of freedom which implies a universal prediction for the subleading corrections to 5 at large distances, which depend only on the number of degrees of freedom of the vibrating string. In the case of the bosonic string in $D$ dimensions one finds [10]:

$$
V(r)=\sigma r+\mu-\frac{\pi(D-2)}{24 r}+\mathcal{O}\left(\frac{1}{r^{2}}\right) .
$$

$\mu$ is some non-universal constant, but the third term, referred to as Lüscher term, is the same for all the string theories in the same universality class.

This prediction could be testable on the lattice formulation of Yang-Mills theory, but this has been an enormous challenge in the past. On the lattice, the static potential is measured from the correlation function of two large Wilson or Polyakov loops (path-ordered products of link variables that loop around the temporal extent of the lattice, see Fig. 3). At large times one can extract the static potential from

$$
\begin{gathered}
\lim _{T \rightarrow \infty}\left\langle P(r) P^{*}(0)\right\rangle=e^{-T V(r)}\left\{1+\mathcal{O}\left(e^{-T \epsilon}\right)\right\} \\
P(r) \equiv \operatorname{Tr}\left[U_{0}(\mathbf{x}, a) U_{0}(\mathbf{x}, 2 a) \ldots U_{0}(\mathbf{x}, T a)\right] .
\end{gathered}
$$

This is however a very difficult measurement because the signal decreases exponentially with the loop area:

$$
\left\langle P(r) P^{*}(0)\right\rangle \sim e^{-\sigma r T},
$$

while the noise is approximately constant. For example, increasing the area of the loop by $1 \mathrm{fm}^{2}$, implies that the 


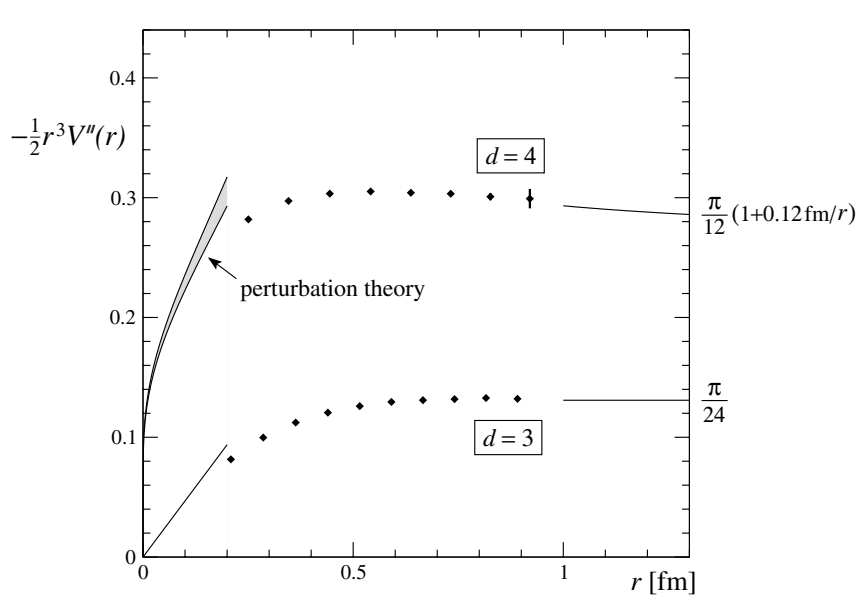

Fig. 4. $-\frac{1}{2} r^{3} V^{\prime \prime}(r)$ as a function of $r$ for $S U(3)$ in $4 \mathrm{D}$ and 3D. The asymptotic solid lines indicate the expectation from the bosonic string. Taken from [13]

statistics has to increase by $3 \times 10^{4}$ to maintain a constant signal/noise!

As a result only in simple gauge theories such as $\mathrm{Z}(2)$ in $3 \mathrm{D}$, where very powerful algorithms are available, has the Lüscher term been computed reliably 11. Recently however the situation has changed, thanks to a new algorithm that can work for any gauge theory 12 . The main idea is to use the locality of the action in order to rewrite average over the product of links (which is the naive definition of the Wilson or Polyakov loops) as a product of the averages of links over smaller sublattices. Since these sublattice averages are exponentially larger than the full average, their numerical evaluation is much simpler. It is now possible to perform calculations of large Polyakov loops at least $O(10-100)$ more precisely and for the first time the Lüscher term has been measured with good accuracy for $S U(3)$ in 3 and 4 dimensions 13 as shown in Fig. 4 It is completely consistent with that expected from the effective bosonic string picture of QCD. In fact the string behaviour sets in at surprisingly short distances around $0.5 \mathrm{fm}$. These results constitute a strong constrain to the dreams of a fundamental equivalence between Yang-Mills and string theory!

\subsection{String breaking from Wilson loops}

The same algorithm mentioned above, has been used to see for the first time the expected string breaking on the lattice from the correlation function of Wilson loops [14.

If you consider the static potential between adjoint charges, the string is expected to break because adjoint charges can be screened by gluons.

The signal of string breaking would be to observe that the static potential stops growing with the distance and remains constant afterwards. This is expected to happen at rather large temporal distances, where algorithms such as the one described above become essential. In fact, for the first time a clear signal of string breaking has been observed in this way [14], as shown in Fig. 5. String breaking

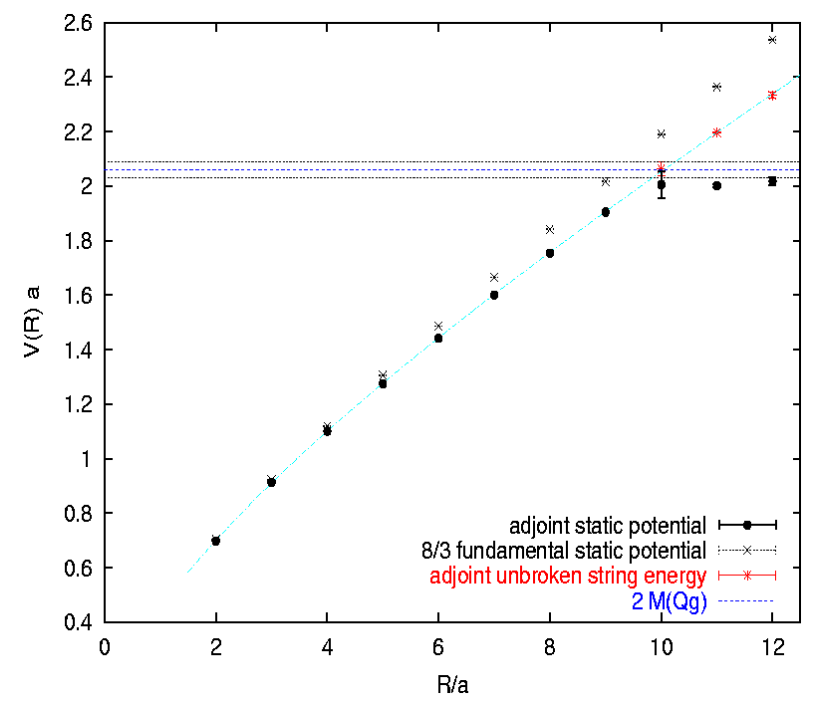

Fig. 5. Static potential between adjoint charges in $S U(2)$ in $3 \mathrm{D}$ as a function of the separation. Taken from 14

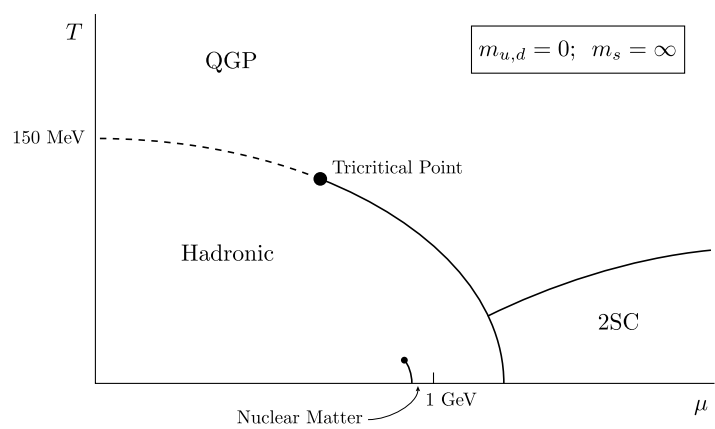

Fig. 6. Conjectured phase diagram of QCD at finite temperature and density

is observed in $S U(2)$ in 3D only at distances of $T \sim 2 \mathrm{fm}$. The potential at that point agrees with twice the minimum energy of the singlet quark-gluon state.

\subsection{QCD at finite density}

The phase diagram of QCD at finite temperature and density has been conjectured to have the rich structure shown in Fig. 6] [15].

The study of this phase diagram on the lattice is very difficult owing to the infamous sign problem, i.e. the fact that the fermion determinant at finite chemical potential, $\mu$, is not positive definite and standard Monte Carlo methods fail.

In the last years, new methods to explore the region of small chemical potential, $\mu_{B}=3 \mu \leq 500 \mathrm{MeV}$, have been proposed. Note that this small $\mu$ region is probably not close enough to reach the tricritical point of Fig. 6, but it encompasses the region relevant for RICH physics, which corresponds to $\mu_{B} \leq 50$. Three different methods have been shown to agree in this regime. They all involve some real approximation to the fermionic action, for which importance sampling can be performed, that can then be 


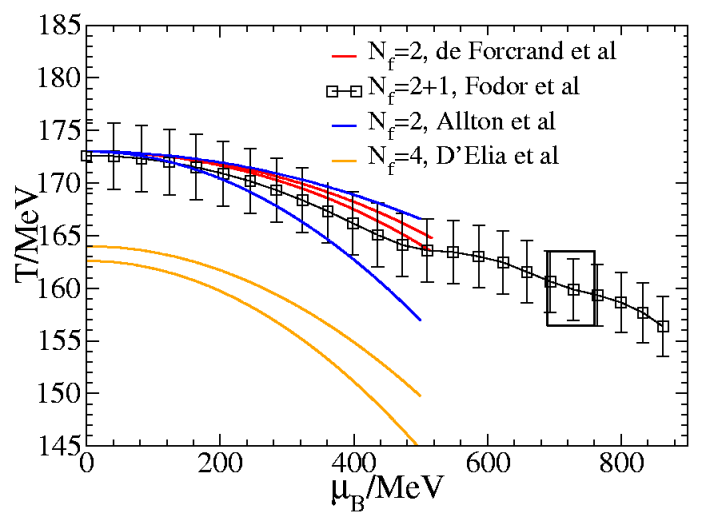

Fig. 7. Comparison of the phase transition line on the plane $(\mu, T)$ with the three methods described in the text. Taken from the second reference in 15

corrected by reweighting the observables. The partition function is rewritten as:

$$
\begin{aligned}
Z[\mu, T] & =\int D\left[A_{\mu}\right] \underbrace{\operatorname{det} \tilde{M} e^{-S_{g}\left[T_{0}\right]}}_{\text {importance sampling }} \underbrace{\frac{\operatorname{det} M(\mu, T) e^{-S_{g}[T]}}{\operatorname{det} \tilde{M} e^{-S_{g}\left[T_{0}\right]}}}_{\text {observable }} \\
& =\left\langle\frac{\operatorname{det}(M(\mu, T)) e^{-S_{g}[T]}}{\operatorname{det}(\tilde{M}) e^{-S_{g}\left[T_{0}\right]}}\right\rangle .
\end{aligned}
$$

where $\operatorname{det} M(\mu, T)$ is the fermion determinant at finite $\mu$ and $T$, while $\operatorname{det} \tilde{M}$ is the real approximation to it. The three methods differ in the definition of $\tilde{M}$ :

- Multiparameter reweighting [16. The idea is to perform the importance sampling with the action at $\mu=0$ and some different temperature $T_{0}$, i.e. $\tilde{M}=M\left(0, T_{0}\right)$. The method seems to be much better behaved than the old idea of using $\tilde{M}=M(0, T)$, because the overlap between the finite $\mu$ and $\mu=0$ ensembles can be enhanced by tunning $T_{0}$ appropriately.

- A Taylor expansion of $M(\mu, T)$ in $\mu / T$ is performed, so that the importance sampling is done with $\tilde{M}=$ $M(0, T)$ [17.

- Importance sampling with imaginary $\mu(\tilde{M}=$ $M(i \mu, T))$ together with an analytic continuation through a Taylor expansion [18.

The results for the critical line of these three different methods are compared in Fig. 7 (taken from the second reference of [15]) for two light flavours. There is good agreement for $\mu_{B} \leq 500 \mathrm{MeV}$, however it is not clear if the tricritical point can be located safely with these methods. The goal of approaching the superconduting phase remains an enormous challenge.

\section{Improved actions}

The idea of Symanzik improvement [19] is that cutoff effects at finite $a$ can be accounted for by the most general continuum Lagrangian including higher order operators compatible with the lattice symmetries:

$$
S_{\text {lat }}(a)=\int d^{4} x\left\{\mathcal{L}_{0}^{d=4}(x)+a \mathcal{L}_{1}^{d=5}(x)+a^{2} \mathcal{L}_{2}^{d=6}(x)+\ldots\right\}
$$

Scaling violations are of $\mathcal{O}(a)$ if there are operators of dimension five which satisfy the lattice symmetries, as for Wilson fermions, or $\mathcal{O}\left(a^{2}\right)$ if they start at dimension six (staggered fermions).

Any observable computed at finite $a$ will show scaling violations of the form:

$$
\operatorname{Observ}(a)=\operatorname{Observ}\left[1+a \Lambda_{1}+\left(a \Lambda_{2}\right)^{2}+\ldots\right] .
$$

The leading scaling violations of $\mathcal{O}(a)$ might come from the operators of dimension five present in the action of (11) or from higher order operators correcting the observable itself. Improvement is the technique to correct for those effects by adding the corresponding operators to the action or the observable and tunning their coefficients to make $\Lambda_{1}$ vanish.

$\mathcal{O}(a)$ improvement has been implemented systematically for Wilson fermions for the action and quark bilinear operators and it has been shown to be very important to keep the continuum extrapolations under control. This was achieved some years ago and good reviews on the subject already exist. We refer the reader to these for further details and references [20]. It should be noted however that partial improvements (for example at tree-level) can make continuum extrapolations more complicated, because subleading $\mathcal{O}\left(a^{2}\right)$ effects can be as large as the uncanceled leading ones, so more parameters are needed in the fits.

The improvement of the weak Hamiltonian (4-fermion operators in $\Delta B, \Delta S$ transitions) remains an enormous challenge. The only hope seems to be to have an exact chiral symmetry on the lattice 1 . In recent years this longstanding goal has has been finally achieved with the socalled Ginsparg-Wilson fermion formulations.

Lattice Dirac operators can be constructed which are local, do not suffer from the doubling problem and satisfy the so-called Ginsparg-Wilson (GW) relation [22]:

$$
\left\{D, \gamma_{5}\right\}=a D \gamma_{5} D .
$$

This relation was first derived for a Dirac operator obtained from a renormalization group blocking of the continuum one. It was found that this relation implies that Ward identities associated with a standard chiral transformation are satisfied on shell at finite lattice spacing, however no explicit solution of (13) was found in [22] and for this reason it fall in oblivion until it was rediscovered in 1997 by P. Hasenfratz 23] in the context of searching for a fixed-point Dirac operator (ie. a fixed-point operator of a renormalization group transformation).

In the meantime, a different avenue was being pursued to search for lattice chirality. It is known since a long time that an infinite domain-wall (DW) in 5D leads naturally to chiral fermions in $4 \mathrm{D}[24,25]$. It was the idea of Ka-

\footnotetext{
1 See however some promising new ideas with twisted-mass Wilson fermions [21.
} 


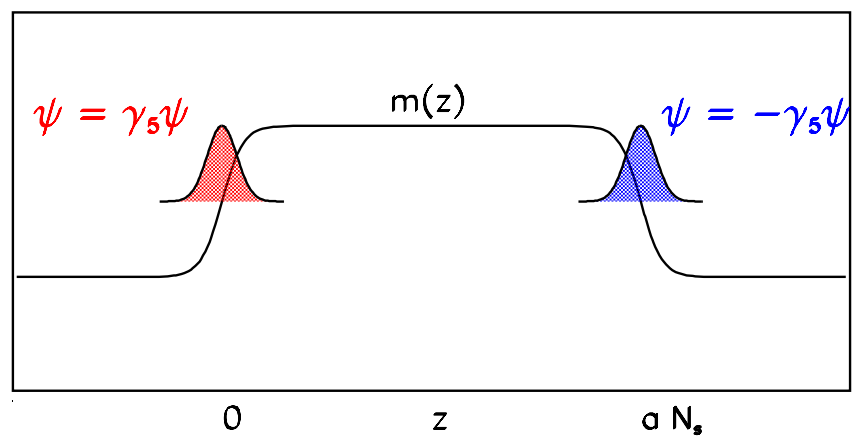

Fig. 8. Finite domain wall

plan 26 27/28] to latticize the DW construction. This requires to introduce a finite lattice spacing in the fourth real dimensions, $a$, and a another one, $a_{s}$ (possibly different), in the extra one. Also a practical implementation requires a finite extent of the extra dimension, with a number of lattice sites $N_{s}$. A five dimensional fermion on this lattice is then coupled to a mass term which varies in the extra dimension as shown in Fig. 8 By analogy with the continuum construction it is expected that if the limit $N_{s} \rightarrow \infty$ is taken at finite $a$ and $a_{s}$, two lattice "chiral" fermions, with opposite chiralities, should appear localized at the two kinks. For a long time it was not clear in what way this lattice construction satisfied a lattice chiral symmetry until it was realized that in fact it is through the magic Ginsparg-Wilson relation.

The effective action and propagator of the light boundary fields can be described in terms of $4 \mathrm{D}$ operator $a D_{N_{s}}$ which satisfies the GW relation in the limit $N_{s} \rightarrow \infty$. This limit can be obtained analytically to give the famous overlap operator [29]:

$$
\begin{aligned}
\lim _{a_{s} \rightarrow 0, N_{s} \rightarrow \infty} a D_{N_{s}} & =a D_{o v}=1+\gamma_{5} \operatorname{sign}(\mathrm{Q}) \\
Q & \equiv \gamma_{5}\left(D_{W}-m_{0}\right)
\end{aligned}
$$

where $D_{W}$ is for example the standard Wilson-Dirac operator and $m_{0}$ is a large mass of $\mathcal{O}\left(a^{-1}\right)$.

$D_{o v}$ was the first explicit construction of GW fermions. It is easy to show that this operator satisfies the GW relation, has the right continuum limit, no doublers and that it is a local operator 30. Indeed it can be shown that the coupling between two fermion fields localized at different sites on the lattice decays exponentially with the distance, $\left\|D_{o v}(x, y)\right\| \leq e^{-\gamma\|x-y\| / a}$.

It was soon realized that in fact the GW relation implies an exact symmetry [31] at finite $a$ under which the fermion and antifermion fields transform in the following way:

$$
\delta_{\chi} \Psi=\epsilon \gamma_{5}(1-a D) \Psi \quad \delta_{\chi} \bar{\Psi}=\epsilon \bar{\Psi} \gamma_{5}
$$

In spite of this exact symmetry, the expected $U_{A}(1)$ anomaly is recovered due to the non-invariance of the fermion measure under a singlet chiral rotation:

$$
\begin{array}{r}
\left\langle\delta_{\chi} O\right\rangle_{F}=\operatorname{Tr}\left[\gamma_{5}(1-a D / 2)\right]\langle O\rangle_{F} \\
=N_{f} \times \operatorname{index}(D)\langle O\rangle_{F}
\end{array}
$$

and an index theorem is exactly satisfied. In particular, this implies that topological sectors can be distinguished [32.

On the other hand the full flavour-symmetry group of QCD can be maintained exactly [33. It is possible to define left and right projectors of the fermion fields:

$$
\Psi_{R, L}=\hat{P}_{ \pm} \Psi \quad \bar{\Psi}_{L, R}=\bar{\Psi} P_{ \pm}
$$

with $P_{ \pm} \equiv\left(1 \pm \gamma_{5}\right) / 2, \hat{P}_{ \pm} \equiv\left(1 \pm \gamma_{5}(1-a D)\right) / 2$ in such a way that the massless fermion action nicely factorizes:

$$
\bar{\Psi} D \Psi=\bar{\Psi}_{L} D \Psi_{L}+\bar{\Psi}_{R} D \Psi_{R} .
$$

It is then trivial to see that there is an exact $S U\left(N_{f}\right)_{L} \times$ $S U\left(N_{f}\right)_{R}$ flavour symmetry under which these chiral projectors transform in a continuum-like fashion:

$$
\Psi_{L} \rightarrow V_{L} \Psi_{L} \quad \Psi_{R} \rightarrow V_{R} \Psi_{R} \quad V_{L, R} \in S U\left(N_{f}\right)_{L, R}
$$

The symmetry can be broken softly by adding quark masses as terms of the form: $\bar{\Psi}_{L} m \Psi_{R}+\bar{\Psi}_{R} m^{\dagger} \Psi_{L}$. The soft breaking then ensures that

- There is a conserved axial current in the naive chiral limit $m \rightarrow 0$

- Operator classification and mixing is enormously simplified: e.g. four fermion operators only mix with those in the same chiral representation

- Scaling violations are $O\left(a^{2}\right)$ : the exact chiral symmetry forbids all operators of $d=5$ in the action. The same is true for many weak composite operators.

GW are expensive to simulate $(\mathcal{O}(100)$ times Wilson), but feasible in the quenched approximation. A number of simulations have been performed recently in realistic lattices of extent $L=1-3 \mathrm{fm}$ with cutoff $a=0.08-$ $0.2 \mathrm{fm}$. In the following, I would like to illustrate the use of these fermions with two recent applications where the exact chiral symmetry has been an essential ingredient.

\section{1 $B_{K}$ with GW fermions}

The measurement of the relevant matrix elements entering $K_{0}-\bar{K}_{0}$ mixing has been an old and great challenge for lattice QCD in the past. The weak Hamiltonian mediating these transitions can be written in terms of $\Delta S=2$ four fermion operators

$$
\mathcal{H}_{\Delta S=2}=C_{W}(\mu) \mathcal{O}_{\Delta S=2}(\mu)+\ldots
$$

which, at the bare level, are products of left currents $\mathcal{O}_{\Delta S=2}^{\text {bare }}=[\bar{s} d]_{V-A}[\bar{s} d]_{V-A}$. The matrix element entering $K_{0}-K_{0}$ mixing is traditionally parametrized in terms of the $B_{K}$ parameter defined as:

$$
\left\langle\bar{K}^{0}\left|\mathcal{O}_{\Delta S=2}(\mu)\right| K^{0}\right\rangle=\frac{8}{3} M_{K}^{2} F_{K}^{2} B_{K}(\mu) .
$$

The computation of these matrix elements on the lattice is not easy with standard fermion regularizations. On 
$\mathrm{B}_{\mathrm{K}}$ from Quenched QCD

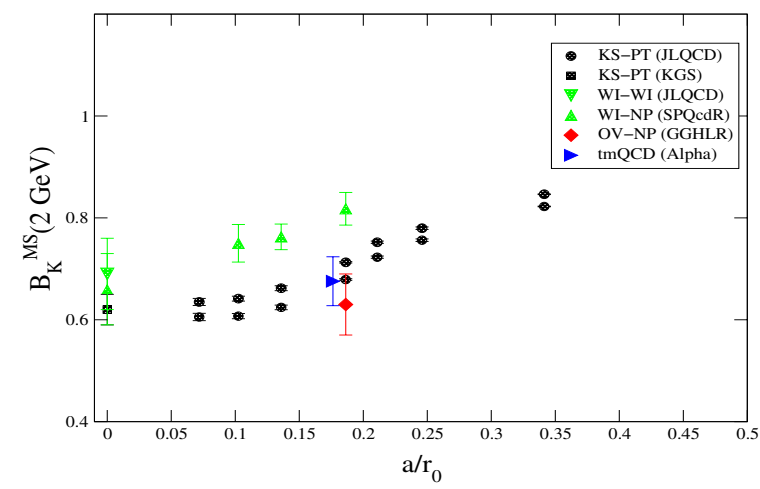

Fig. 9. $B_{K}^{M S}(2 G e V)$ in several computations using different fermion formulations as a function of the lattice spacing in units of $r_{0}=0.5 \mathrm{fm}$. The GW determination (diamond) of [36] in a rather coarse lattice is in good agreement with the continuum results using staggered (square and circle) and Wilson fermions (triangles in all orientations). Taken from [5]

the one hand, in the case of Wilson fermions, the breaking of chiral symmetry implies that the bare operator mixes with other chiral structures $(V \times V-A \times A, S \times S \pm P \times P$, $T \times T)$ which makes the non-perturbative renormalization extremely complicated in practice. Only recently smart tricks have been found to get rid of these mixings 34 . In the case of staggered fermions, a subgroup of the chiral group is maintained but at the cost of breaking the flavour group. Mixing with many other flavour structures occurs and the renormalization has only been done perturbatively in this case [35. Instead with GW fermions the renormalization is as simple as in the continuum: it is just multiplicative. Even though Wilson fermions are presently also able to perform the renormalization of these operators non-perturbatively, the scaling violations in that case are of $\mathcal{O}(a)$, while they are suppressed to $\mathcal{O}\left(a^{2}\right)$ in the case of GW fermions, which makes the new determination of $B_{K}$ of 36 competitive as shown in Fig. 9] For more details see the parallel contribution in 5 .

\subsection{QCD versus random matrix theory}

In the presence of spontaneous chiral symmetry breaking $(\chi \mathrm{SB})$, the QCD partition function at fixed topological charge has been conjectured to be equivalent to that of a Random Matrix Theory (RMT) [37]. For a review see [38. More precisely if the infinite volume limit of the QCD partition function at fixed topological charge $\nu$ is taken keeping the quantity $m \Sigma V$ fixed, where $\Sigma$ is the quark condensate, then:

$$
\begin{array}{r}
\lim _{N \rightarrow \infty, m \rho(0) N=\text { fixed }} Z_{\nu}^{R M T}(m)= \\
V \rightarrow \infty, m \Sigma V=\text { fixed } \\
\lim _{\nu}^{Q C D}(m)
\end{array}
$$

where the RMT partition function is:

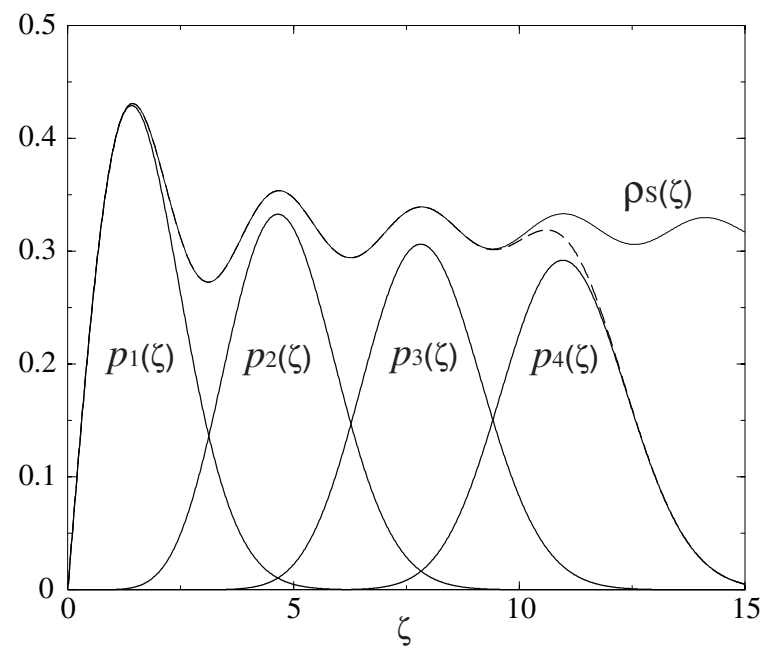

Fig. 10. $\rho(\lambda)$ of the Dirac operator

$$
Z_{\nu}^{R M T} \equiv \int d W \prod_{f=1}^{N_{f}} \operatorname{det}(i M+m) \exp \left[-N / 2 \operatorname{Tr} V\left(M^{2}\right)\right]
$$

with $M=\left(\begin{array}{cc}0 & W^{\dagger} \\ W & 0\end{array}\right)$, and $W$ is a random complex matrices of rectangular size $N \times(N+\nu)$. $\rho(\lambda)$ is the density of eigenvalues of $M$ close to zero. Note that the limit on the left-hand side of (22), or microscopic limit, implies to take the size of the matrices, $N$, to infinity keeping $m \rho(0) N$ fixed and it assumes that the eigenvalue density close to zero is finite, $\rho(0) \neq 0$. In this case, it well known that the eigenvalue spectrum close to zero is universal for all these RMT, that is independent of $V(M)$.

This equivalence gives a precise prediction for the eigenvalue density close to zero as well as for the individual low-lying eigenvalue distributions of the Dirac operator. The eigenvalue density is given in terms of Bessel functions and depends only on the condensate, $\Sigma$ :

$$
\begin{aligned}
\rho_{\nu}(\lambda) & =\frac{\Sigma \zeta}{2}\left(J_{N_{f}+\nu}(\zeta)^{2}-J_{N_{f}+\nu-1}(\zeta) J_{N_{f}+\nu+1}(\zeta)\right) \\
\zeta & \equiv \lambda \Sigma V
\end{aligned}
$$

and is depicted in Fig. 10

In order to test this prediction on the lattice, it is essential to have an exact chiral symmetry and distinguish topological sectors. Previous simulations with staggered fermions 38] have shown clear evidence in favour of the conjecture, however staggered fermions are not in the same universality class as QCD at finite lattice spacing. Only recently this conjecture has been tested close to the continuum limit and in realistic volumes using GW fermions, which are indeed in the same universality class as $\mathrm{QCD}(S U(3)$ with matter in the fundamental) as shown in Fig. 11$]$ 39.

The agreement of the ratios of the averages of individual eigenvalues with the predictions of RMT is truly 


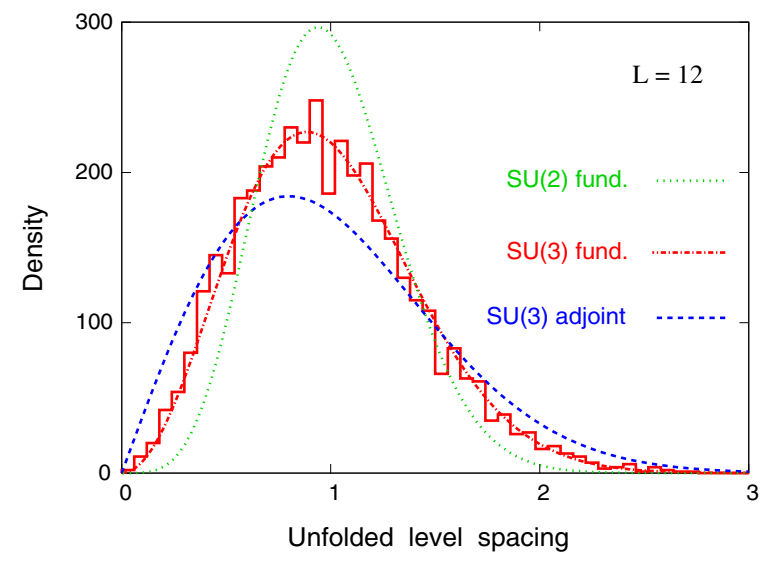

Fig. 11. Unfolded level spacing measured (histogram) compared with the predictions of RMT for the three RMT universality classes. Taken from 39]

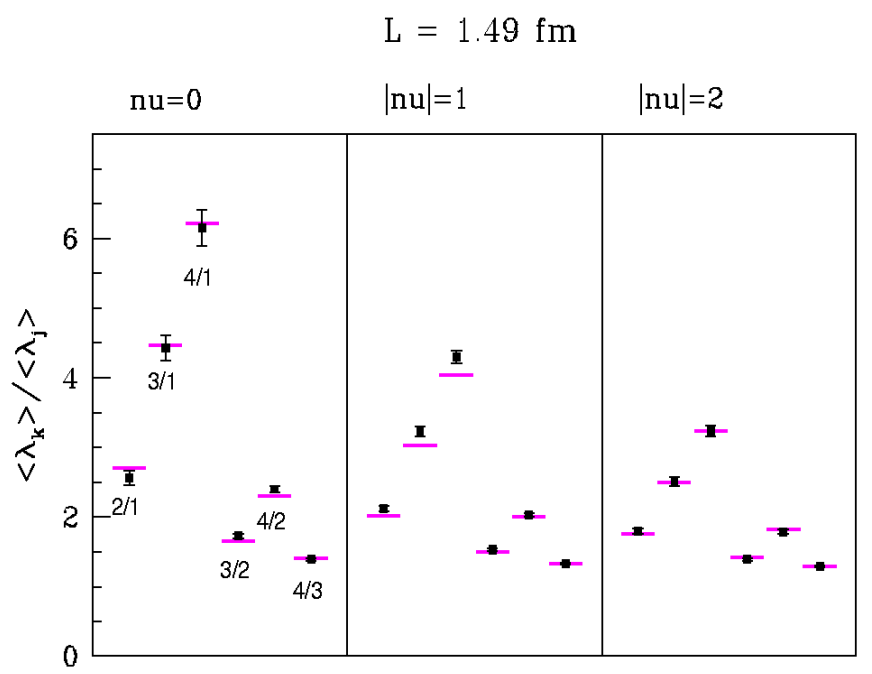

Fig. 12. Ratio of averages of eigenvalues measured in quenched QCD in a lattice $L=1.49 \mathrm{fm}$ and $a=0.074 \mathrm{fm}$ for the three topological sectors with charge $\nu=0,1,2$. Taken from [40]

remarkable for $L \geq 1.2 \mathrm{fm}$ [40], as shown in Fig.[12, where the ratio of the averages of the different eigenvalues are compared with the parameter-free predictions of RMT.

The averages themselves can also be compared with the RMT predictions, which depend only on one low energy coupling: $\Sigma$, the chiral condensate. In fact this method gives not only the most precise determination of $\Sigma$, but also a very clean proof that indeed this is the order parameter of chiral symmetry breaking in QCD.

\section{Ask the right questions}

A typical problem in lattice QCD is the two or multi-scale problem as depicted in Fig. 13, that is the fact that there is more than one relevant scale. In this case, a realistic lattice simulation requires very large lattices, because it is necessary to ensure that the wavelength of the heavy scale is large compared to the lattice spacing, $\lambda_{\text {heavy }} / a \gg 1$,
Two-scale problem

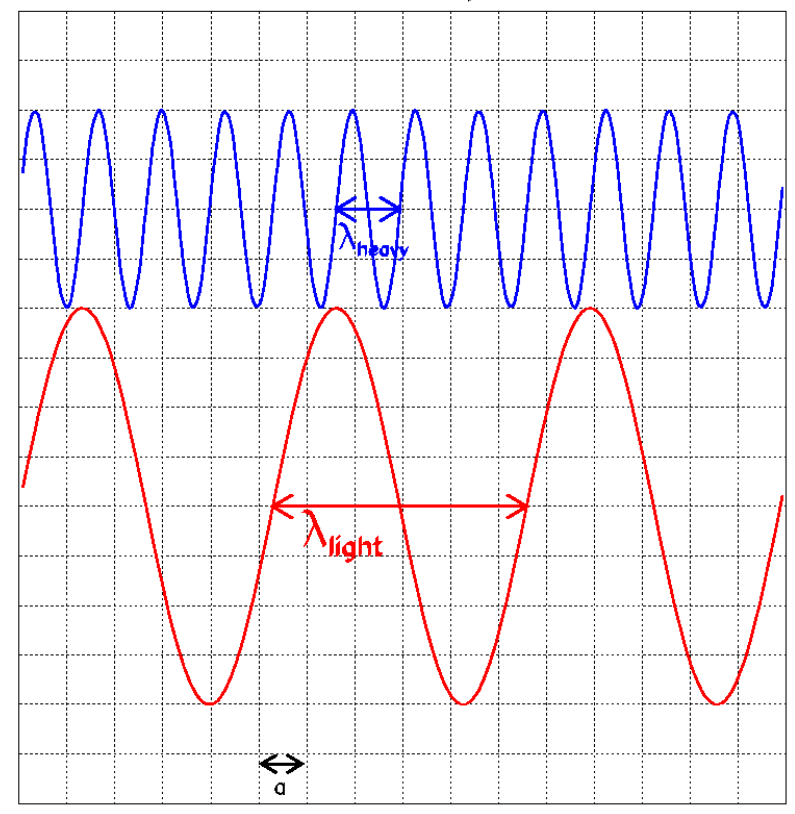

Fig. 13. Two-scale problem

to avoid large cutoff effects, but on the other hand the wavelength of the light scale should be large compared to the lattice size, $\lambda_{\text {light }} / L \ll 1$, since otherwise large finite size effects will be present. The result is that the number of points in the lattice, $L / a$, has to be increased by a factor $\sim \frac{\lambda_{\text {light }}}{\lambda_{\text {heavy }}}$ with respect to the one-scale problem.

This situation is quite common in realistic situations both in heavy quark physics where $\lambda_{\text {light }} \sim \Lambda_{Q C D}^{-1} \gg$ $\lambda_{\text {heavy }} \sim m_{b}^{-1}$ as in light quark physics where $\lambda_{\text {light }} \sim$ $m_{\pi}^{-1} \gg \lambda_{\text {heavy }} \sim \Lambda_{Q C D}^{-1}$. Some important progress has been achieved recently by designing methods to solve in a more efficient way the multi-scale problem, both in heavy and light quark physics.

\subsection{Heavy quark physics}

The lattice can in principle provide precise numbers for many quantities of phenomenological interest such as the B meson decay constants and bag parameters:

$$
F_{B, B_{s}}, B_{B, B_{s}}, \xi \equiv \frac{F_{B_{s}} \sqrt{B_{B_{s}}}}{F_{B_{d}} \sqrt{B_{B_{d}}}}
$$

where

$$
\begin{array}{r}
\left\langle B_{q}\left|\mathcal{O}_{\Delta M=2}\right| B_{q}\right\rangle=\frac{4}{3} m_{B_{q}}^{2} F_{B_{q}}^{2} B_{B_{q}} \\
\left\langle B_{q}\left|\bar{b} \gamma_{\mu} \gamma_{5} q\right| 0\right\rangle=i p_{\mu} F_{B_{q}}
\end{array}
$$

Many results have been obtained in recent years using a plethora of approximations to overcome the two-scale problem. Unfortunately, errors are mostly dominated by 

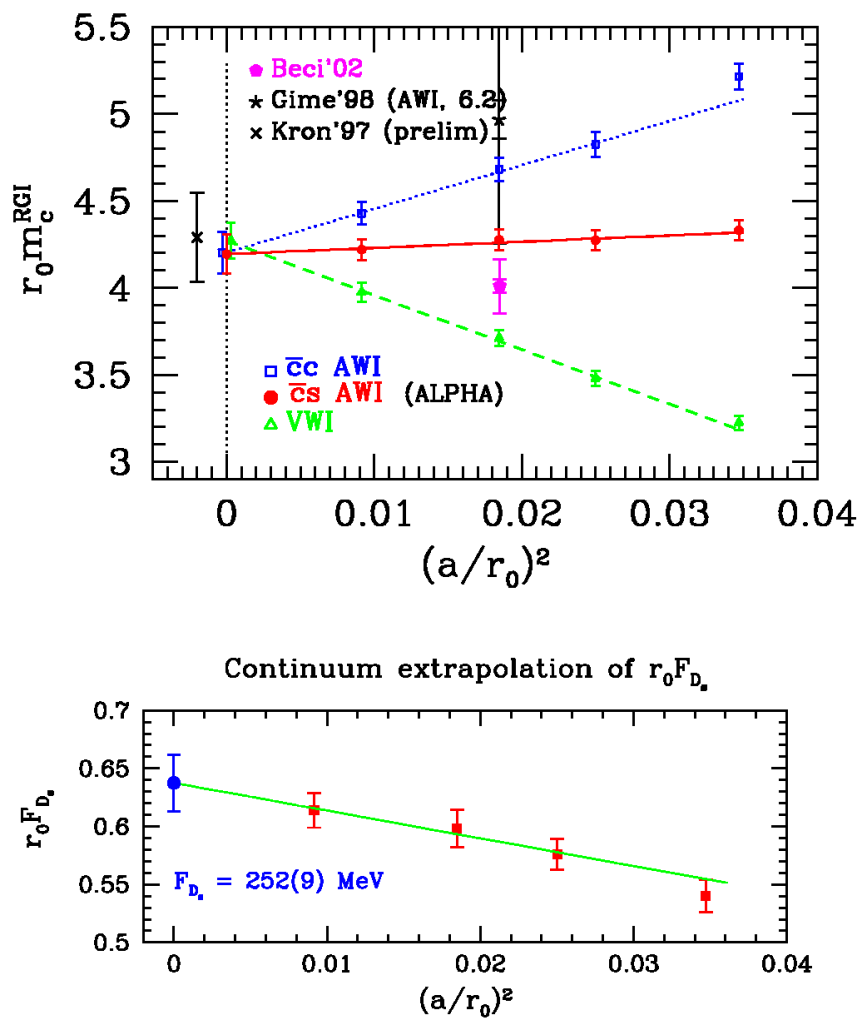

Fig. 14. Up: continuum extrapolation of the charm quark mass using three different methods and $\mathcal{O}(a)$-improved Wilson fermions (from 41]). Down: continuum extrapolation of the $D_{s}$ meson decay constant using $\mathcal{O}(a)$-improved Wilson fermions (from 42)

systematics associated to these approximations. There are essentially two strategies:

Class A: use relativistic heavy quarks

It has been demonstrated that typical lattice sizes can reliably accommodate quarks in the charm region using $\mathcal{O}(a)$-improved Wilson fermions. In Fig.14, the continuum extrapolation of the charm quark mass and the $D_{s}$ meson decay constant in the quenched approximation are shown. Clearly the cutoff effects are under control.

The charm quark mass and $F_{D_{s}}$ decay constant have thus been obtained in the quenched approximation with unprecedent precision (systematic errors other than quenching effects are fully understood):

$$
m_{c}^{\overline{M S}}\left(m_{c}^{\overline{M S}}\right)=1.301(34) \quad F_{D_{s}}=252(9) \mathrm{MeV} .
$$

However, these results also indicate that, given the size of the cutoff effects in Fig. 14, dangerously large extrapolations would be needed for quark masses in the $b$-quark mass region.

The hyperfine splitting in charmonium has also been computed very precisely using $\mathcal{O}(a)$-improved Wilson fermions 43 . This splitting is shown in Fig. 15 for fully improved, tree-level improved and unimproved Wilson fermions. Clearly full non perturbative improvement is very important to have a reliable continuum extrapolation as is illustrated by the different results from the different
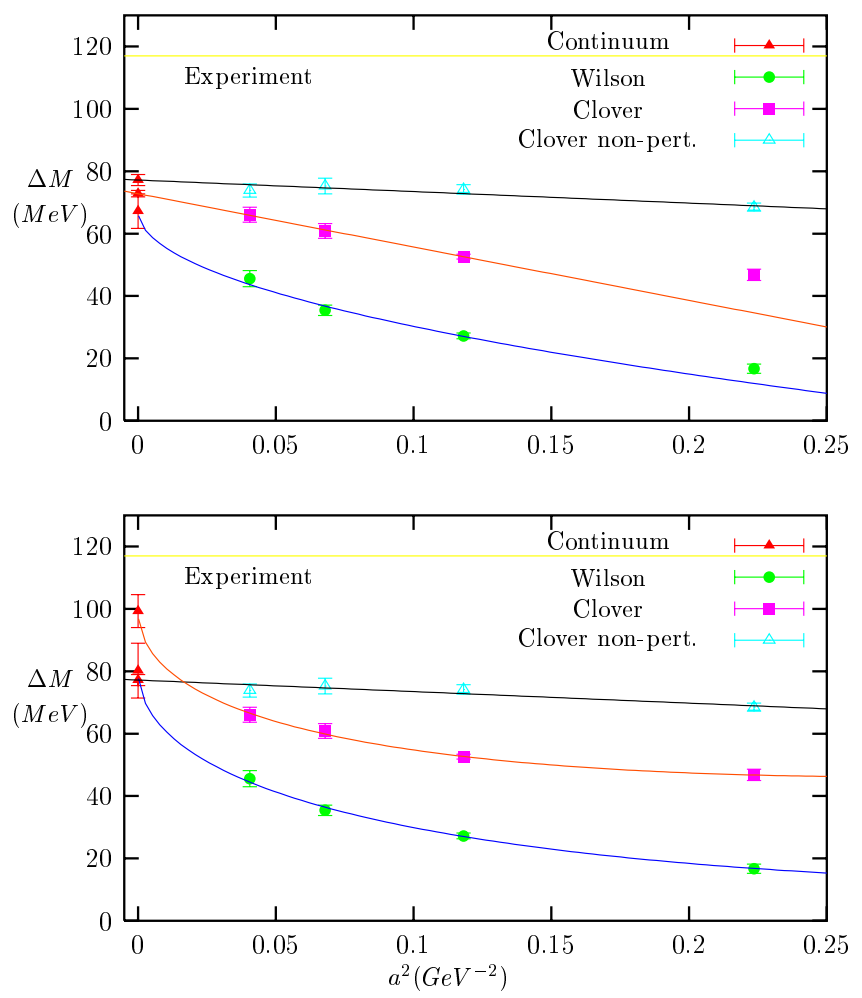

Fig. 15. Continuum extrapolation of the hyperfine splitting of charmonium. Data are shown for tree-level improved (Clover), non-perturbatively improved (Clover non-pert.) and unimproved Wilson fermions (Wilson). Two different fits for the tree-level improved and unimproved data are shown: linear in $a^{2}$ and $a$ respectively ( $u p$ ) and quadratic (with $a$ and $a^{2}$ terms) (down). Taken from 43

fits to the unimproved or tree-level improved data. The continuum value obtained for $\Delta M=M(J / \psi)-M\left(\eta_{c}\right)=$ $77(2)(6) \mathrm{MeV}$ is considerably smaller than the experimental value $117 \mathrm{MeV}$. The difference must be due to the quenched approximation. Interestingly this is one of the quantities which shows largest quenching artifacts.

Class B: non-relativistic expansion in $\frac{\Lambda_{Q C D}}{m_{b}}$

It is well-known that a very useful method in $b$-physics is the heavy quark effective theory (HQET) [46], in which the degrees of freedom associated with the $b$-quark mass are integrated out. At order $\mathcal{O}\left(1 / m_{b}\right)$, the Lagrangian is given by:

$$
\begin{aligned}
\mathcal{L}_{H Q E T}=\bar{\Psi}_{h} D_{0} \Psi_{h}-\frac{1}{2 m_{b}} \bar{\Psi}_{h} \mathbf{D}^{2} \Psi_{h} & -\frac{c_{\sigma}}{2 m_{b}} \bar{\Psi}_{h} B \cdot \sigma \Psi_{h} \\
& +\mathcal{O}\left(\frac{\Lambda_{Q C D}}{m_{b}}\right)^{2}
\end{aligned}
$$

The idea here is to use this effective theory on the lattice since the cutoff can then be much lower than $m_{b}$. The main difficulty of this method comes however from the fact that the matching (the determination of the couplings in the effective theory) and the renormalization of the effective theory must be done non-perturbatively, because there are generically power divergences [4]. If the matching is for 
example done perturbatively at $l$-th loop level, an error in the coupling $c_{k}$ remains:

$$
\Delta c_{k} \sim \frac{g_{0}^{2(l+1)}}{a} \sim \frac{1}{\operatorname{aln}(a \Lambda)^{l+1}} \stackrel{a \rightarrow 0}{\longrightarrow} \infty,
$$

and the continuum limit strictly speaking cannot be taken.

The second difficulty comes from the fact that it is only realistic to use HQET at NLO (since higher order involve many more couplings). Working at this order implies that uncertainties $\mathcal{O}\left(\frac{\Lambda_{Q C D}}{m_{b}}\right)^{2}$ remain. Nevertheless it is still very useful since the static limit can be combined with the relativistic approach to perform an interpolation to $m_{b}$ as opposed to an extrapolation from either end. Also the non-perturbative determination of the couplings and matrix elements in HQET gives very valuable information for continuum phenomenology.

In the past year, significant progress has been achieved in class A and B methods to reduce systematic errors with the use of finite-size scaling techniques.

Recently it has been realized that the $b$-quark can be simulated in a small volume, such that $L_{0} m_{b} \gg 1, m_{b} a \ll$ $1, L_{0} \Lambda_{Q C D} \leq 1$ [44, 45]. This of course implies that any observable will show large finite volume effects. However these can be corrected by multiplying the observable by a step scaling function, which relates the observable computed in the small volume to that in a volume twice as large:

$$
\sigma\left(m_{h}, m_{l}, L_{0}\right) \equiv \frac{\mathcal{O}\left(m_{h}, m_{l}, 2 L_{0}\right)}{\mathcal{O}\left(m_{h}, m_{l}, L_{0}\right)}
$$

and depends on the volume and the light and heavy scales. The process can be iterated to arrive to a realistically large volume:

$$
\mathcal{O}\left(m_{h}, m_{l}, 2^{n} L_{0}\right)=\prod_{i=1}^{n-1} \sigma\left(m_{h}, m_{l}, 2^{i} L_{0}\right) \mathcal{O}\left(m_{h}, m_{l}, L_{0}\right)
$$

The important observation is that the step scaling function has a continuum limit, which can taken at each step, and that it is a much smoother function of the heavy mass, $m_{h}$, since the leading dependence on this scale, $\mathcal{O}\left(m_{l} / m_{h}\right)$, is expected to cancel in the ratio so that only corrections of $\mathcal{O}\left(1 / m_{h} L\right)$ remain in $\sigma$. The series of step functions can be computed in a series of lattices with increasing $a$ and $L_{0}$ and roughly fixed $a m_{h}$ and $m_{h} L_{0}$. A continuum and heavy quark mass extrapolations are performed at each step.

Figure [16] shows the heavy quark mass extrapolation of the scaling function of the heavy-light decay constants. The extrapolation to $m_{b}$ in the largest volume is a $6 \%$ effect as opposed to a $20 \%$ effect in the standard relativistic approach.

With this method, new results for the B and D meson decay constants, $F_{B, B_{s}}, F_{D, D_{s}}$ as well as the $b$-quark

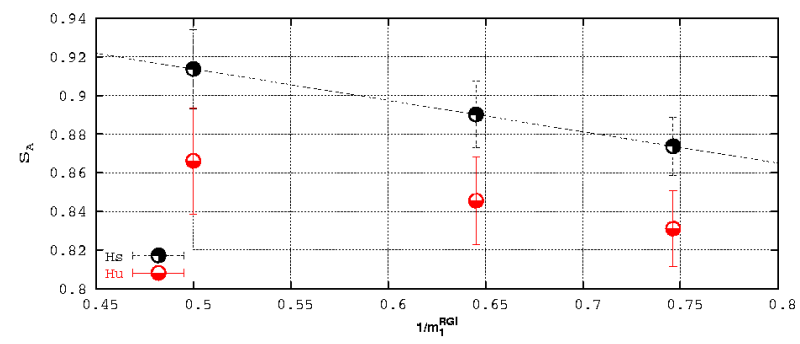

Fig. 16. Heavy quark mass extrapolation to the $b$-quark mass of the step scaling function of the heavy-strange $(H s)$ and heavy-light $(\mathrm{Hu})$ decay constants. Taken from 45]

mass have been computed with significantly smaller systematic uncertainties (other than quenching). A summary plot from the parallel contribution [9] compares these results with previous ones in Fig. 17

Concerning class $\mathrm{B}$ methods, in the past year a new method has been proposed to perform the nonperturbative matching and renormalization of HQET using again finite-size scaling techniques 48 49. The main idea is to match QCD and HQET in a finite (small) volume and then use an iterative procedure, to run to $L \rightarrow \infty$ within the effective theory, with a cutoff $a^{-1} \ll m_{b}$.

One of the examples considered by the authors of 49 is that of the $b$-quark mass in the static limit. The starting point is the computation of the energy of a state with the quantum numbers of the B meason, $\Gamma\left(L_{0}\right)$, in a finite volume $V=L_{0}^{4}$ in QCD. In order to correct for finite-size effects, the difference between this quantity measured in the effective theory in a lattice with size $L_{0}$ and $2 L_{0}$ is computed and extrapolated to the continuum limit. This computation is repeated a number of times in order to approach eventually a realistic volume size. As the volume is increased, the cutoff can be increased so that the number of points in the lattice is roughly the same in each iteration. The final value of the $b$-quark mass can the be obtained from:

$$
\begin{aligned}
\underbrace{M_{B}}_{\text {exp. }} & =\underbrace{\Delta \Gamma_{\text {stat }}\left(2^{2} L_{0}, a\right)}_{a<0.07 f m \rightarrow 0}+\underbrace{\Delta \Gamma_{\text {stat }}\left(2 L_{0}, a\right)}_{a<0.05 f m \rightarrow 0} \\
& +\underbrace{\Delta \Gamma_{\text {stat }}\left(L_{0}, a\right)}_{a<0.025 f m \rightarrow 0}+\underbrace{\Gamma_{Q C D}\left(L_{0}, m_{b}\right)}_{a<0.0125 f m \rightarrow 0}
\end{aligned}
$$

with $\Delta \Gamma_{\text {stat }}(L, a) \equiv \Gamma_{\text {stat }}(2 L, a)-\Gamma_{\text {stat }}(L, a)$.

The result for the $b$-quark mass in the static limit reported in the last reference of 49 is

$$
m_{b}^{\overline{M S}}\left(m_{b}^{\overline{M S}}\right)=4.12(8) \mathrm{GeV}+\mathcal{O}\left(\frac{\Lambda}{m_{b}}\right)
$$

More recently results for $F_{B_{s}}$ have been presented in 50. from an interpolation of the renormalization group invariant axial current matrix element between the relativistic and static result as shown in Fig. 18

$$
F_{B_{s}}=206(10) \mathrm{MeV}
$$



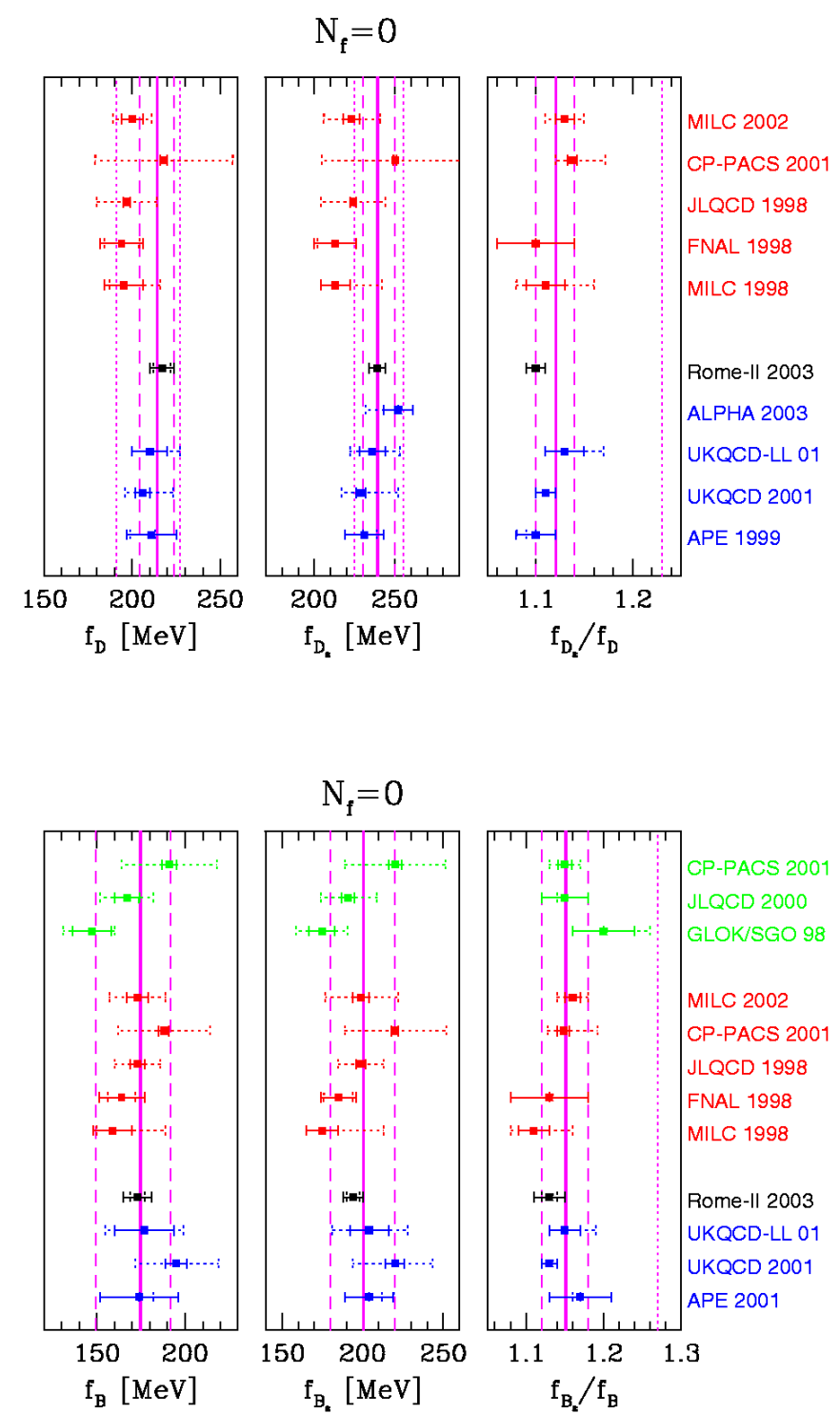

Fig. 17. Summary plots for the $D$ and $B$ mesons decay constants, taken from [9]. The points labeled Rome-II correspond to the finite size scaling approach discussed in the text

For more details on this method, see the parallel contribution in 6 .

Unfortunately, these promising ideas have not yet been applied to the matrix elements of four-fermion operators. Some interesting new results on the determination of lifetime ratios and width differences were presented in the parallel contribution of 8$]$.

\subsection{Light quark physics}

The approach to the regime of the light quark masses is one of the most difficult problems in Lattice QCD both quenched and unquenched. Typical simulations consider quark masses larger than $\frac{m_{s}}{2}$. The difficulty in approaching the regime of the light quark masses originates in the vacuum structure of QCD. Spontaneous chiral symmetry

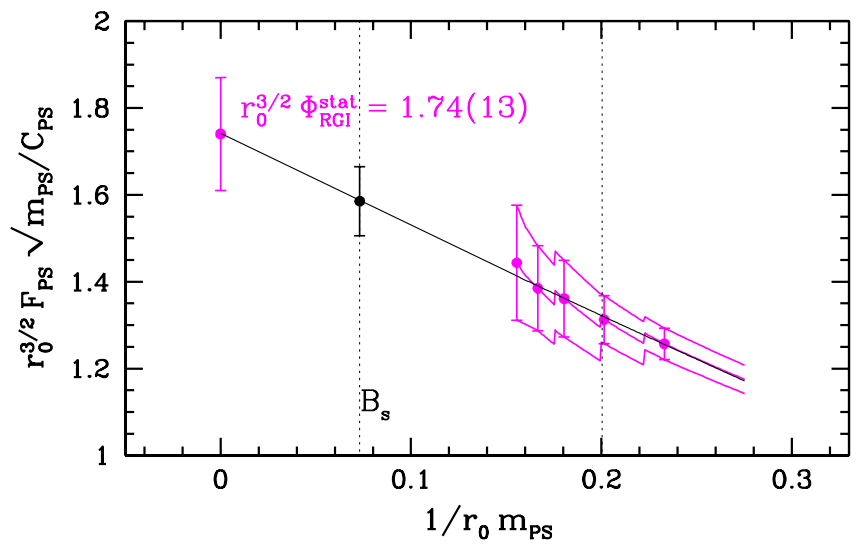

Fig. 18. Interpolation of the renormalization group invariant matrix element of the axial current (which is proportional to the decay constant) between the static limit and the relativistic determinations around the charm quark mass. Taken from 6

breaking $(\chi \mathrm{SB})$ via a quark condensate implies that the density of eigenvalues close at zero is finite [51]:

$$
\lim _{m \rightarrow 0}\langle\bar{q} q\rangle \equiv-\Sigma=-\pi \rho(0) \neq 0 .
$$

In a finite volume the implication is that both the low (non-zero) eigenvalues and also the level splittings are controlled by the parameter $\frac{1}{\Sigma V}$ [52], so the lowest eigenvalue in particular decreases linearly with the volume. This poses on the one hand an algorithmic problem in the quenched case, where the cost of computing a quark propagator increases linearly with the volume close to the chiral limit, that becomes much more serious in the unquenched case. Furthermore, the absence of a gap in the spectrum implies a two-scale problem in that the two different scales: the pseudoscalar mass and $\Lambda_{Q C D}$ have to be treated simultaneously.

The standard approach to extract the physics of light quarks is to use $\chi \mathrm{PT}$ to extrapolate results from $m_{q} \simeq$ $m_{s} / 2 \rightarrow 0$. However it has been shown that this is the source of one of the most important systematic uncertainties both in light (e.g. $F_{\pi}$ ) and heavy quark physics (e.g. $F_{B_{d}}, B_{B_{d}}$ ). In Fig. 19 the chiral extrapolations of the pseudoscalar decay constant for light-light, $F_{P S} r_{0}$, and heavy-light mesons, $F_{B_{q}} \sqrt{M_{B_{q}}}$, are shown. The systematic uncertainty in the chiral extrapolations is clearly very large.

The combination of the lattice and $\chi \mathrm{PT}$ is in principle the most economical way of studying the physics of light mesons at low momenta, since these are determined to a great extent by the pattern of chiral symmetry breaking. The QCD $\chi$-Lagrangian incorporates automatically all the Ward identity relations and parametrizes what is not determined by symmetry by a set of low energy constants [54]. An expansion at low momenta gives the following Lagrangian:

$$
\mathcal{L}_{\chi}^{Q C D}=\mathcal{L}^{(2)}+\mathcal{L}^{(4)}+\ldots
$$

$\mathcal{L}_{\chi}^{(2)}=\frac{F^{2}}{4} \operatorname{Tr}\left[\partial_{\mu} U^{\dagger} \partial_{\mu} U\right]-\frac{\Sigma}{2} \operatorname{Tr}\left[e^{i \theta / N_{f}} M U+\right.$ h.c. $]$ 

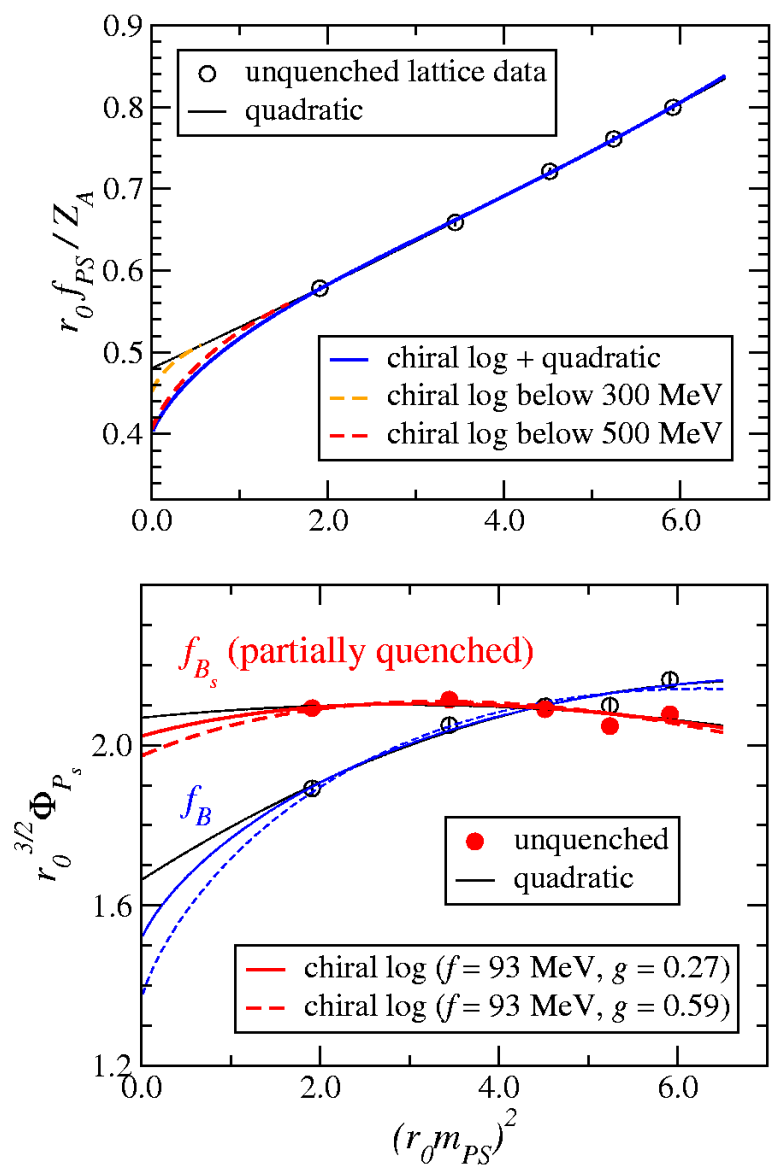

Fig. 19. Chiral extrapolations of the pseudoscalar decay constant (up) and that of the $B_{s}$ and $B$ mesons (down) with partially quenched $\left(N_{f}=2\right)$ lattice data. Taken from [53]

$$
\mathcal{L}_{\chi}^{(4)}=L_{1} \operatorname{Tr}\left[\partial_{\mu} U^{\dagger} \partial_{\mu} U\right]^{2}+L_{2}\left(\operatorname{Tr}\left[\partial_{\mu} U^{\dagger} \partial_{\nu} U\right]\right)^{2}+\ldots
$$

with $M$ the quark mass matrix, $U=e^{i 2 \Phi / F}, \Phi$ the light meson field and $\theta$ the vacuum angle.

The low energy couplings of this Lagrangian (LECs): $\Sigma, F, L_{i=1-10}, \ldots$ parametrize the non-perturbative dynamics that is not determined by symmetry and the lattice is the optimal method to determine them.

The weak interactions responsible for weak decays such as $\Delta S=1$ can also be included in the effective theory:

$$
\mathcal{L}^{\Delta S=1}=\frac{G_{F}}{2 \sqrt{2}} \sin \theta_{C} \cos \theta_{C} \sum_{i} C_{W}^{i}(\mu) O_{i}(\mu)
$$

where the $O_{i}^{\prime} s$ transform as $(27,1),(8,1)$ or $(8,8)$ under $S U(3)_{L} \times S U(3)_{R}$ : to leading order there are only four such operators:

$$
\begin{aligned}
\mathcal{L}_{\chi}^{\Delta S=1} & =g_{(27,1)} t_{k l}^{i j}\left(U \partial_{\mu} U^{\dagger}\right)_{i k}\left(U \partial_{\mu} U^{\dagger}\right)_{j l} \\
& +g_{(8,8)} \tilde{t}_{k l}^{i j} U_{i k} U_{j l}^{\dagger}+g_{(8,1)}^{(1)} c_{i j}\left(\partial_{\mu} U \partial_{\mu} U^{\dagger}\right)_{i j} \\
& +g_{(8,1)}^{(2)} c_{i j}\left(M U+U^{\dagger} M^{\dagger}\right)_{i j}+\ldots,
\end{aligned}
$$

where $t_{k l}^{i j}, \tilde{t}_{k l}^{i j}$ and $c_{i j}$ are apropriate tensors.
Table 1. Phenomenological determination of the LECs [55]

\begin{tabular}{|c|c|c|}
\hline$i$ & $\alpha_{i}^{r}\left(M_{\rho}\right) \equiv 8(4 \pi)^{2} L_{i}^{r}\left(M_{\rho}\right)$ & $O\left(N_{C}\right)$ \\
\hline $2 \alpha_{1}-\alpha_{2}$ & $-0.8 \pm 0.8$ & $O(1)$ \\
$\alpha_{2}$ & $1.7 \pm 0.4$ & $O\left(N_{C}\right)$ \\
$\alpha_{3}$ & $-4.4 \pm 1.4$ & $O\left(N_{C}\right)$ \\
$\alpha_{4}$ & $-0.4 \pm 0.6$ & $O(1)$ \\
$\alpha_{5}$ & $1.8 \pm 0.6$ & $O\left(N_{C}\right)$ \\
$\alpha_{6}$ & $-0.25 \pm 0.4$ & $O(1)$ \\
$\alpha_{7}$ & $-0.5 \pm 0.25$ & $O(1)$ \\
$\alpha_{8}$ & $1.1 \pm 0.4$ & $O\left(N_{C}\right)$ \\
$\alpha_{9}$ & $8.7 \pm 0.9$ & $O\left(N_{C}\right)$ \\
$\alpha_{10}$ & $-6.9 \pm 0.9$ & $O\left(N_{C}\right)$ \\
\hline
\end{tabular}

The problem is that the matching between the effective theory and QCD to fix the low-energy couplings should be done at $m_{q} \rightarrow 0$ and not at $m_{q} \rightarrow m_{s}$, because higher order effects may be important around $m_{s}$ and it is not clear how many couplings are necessary in this range. This matching is however an essential step to check the range of validity of $\chi \mathrm{PT}$ and to get rid of the systematic error in chiral extrapolations.

Obviously getting the low energy couplings, which are presently rather poorly constrained from phenomenology (see Table 1), would be extremely important for many applications. For example the fundamental question of whether $m_{u}=0$ or not is connected to the value of $2 \alpha_{8}-\alpha_{5}$, which cannot be determined unambiguously in $\chi \mathrm{PT}$ [56]. This combination can be measured unambiguously on the lattice and this has been done recently both in the quenched and partially quenched approximations. The results are summarized in Fig. 20.

There is a good statistical accuracy in this quantity, but there are still large systematic effects coming from chiral extrapolations, as discussed above. On the other hand, an unreasonably large unknown systematic effect would be needed to accommodate $m_{u}=0$.

Recently the goal of matching QCD and $\chi \mathrm{PT}$ at $m_{q} \rightarrow$ 0 is being pursued actively. The main ingredients are on the one hand the use of GW fermions, which satisfy an exact chiral symmetry, and on the other the use of finitesize scaling techniques to avoid the two-scale problem. In particular, the chiral limit can be taken in a finite volume. Provided $L \Lambda_{Q C D} \gg 1$, finite volume effects are fully calculable within $\chi \mathrm{PT}$ in terms of the infinite-volume low energy constants. Lattice simulations are always done in a reasonably large volume compared to $\Lambda_{Q C D}$. However depending on the quark mass, one can encounter the situations illustrated in Fig. 21 When the volume and the quark mass are such that the Compton wavelength of the pion is much shorter than the size of the box, $m_{\pi} L \gg 1$, finite volume effects are exponentially supressed. See left plot in Fig. [21, On the other hand if the quark mass is further decreased at some point $m_{\pi} L \sim 1$ and finite-size corrections are suppressed like a power $\mathcal{O}\left((F L)^{-2}\right)$ (Fig. 21 

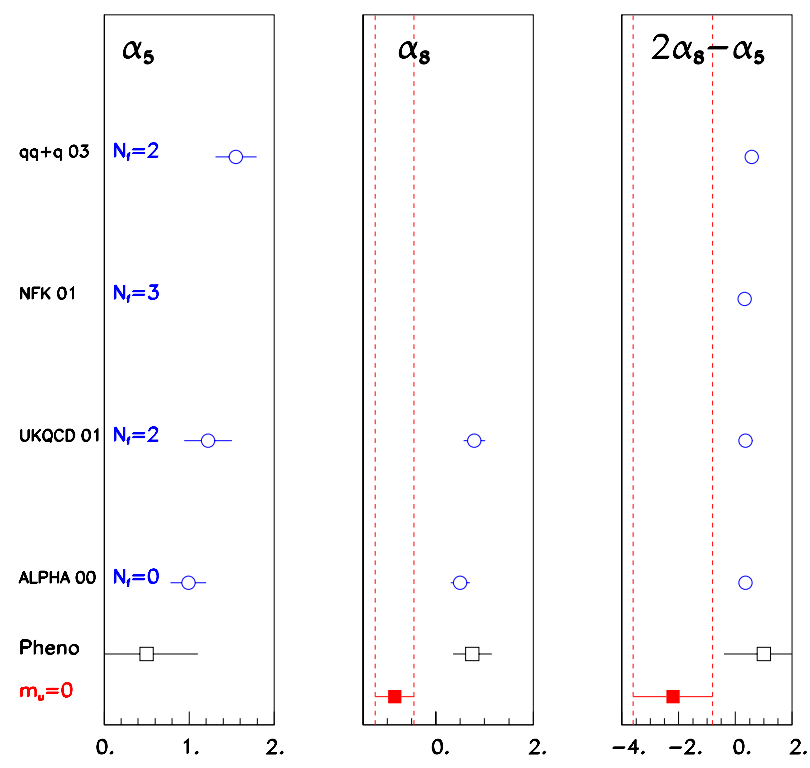

Fig. 20. Lattice determinations of the couplings $\alpha_{5}, \alpha_{8}$ and $2 \alpha_{8}-\alpha_{5}$ for quenched [57] and partially quenched [58,59,60. data as indicated by $N_{f}$. The full squares and their associated vertical bands are the expected values that would be compatible with the hypothesis $m_{u}=0$, while the open squares are the values favoured phenomenologically by large $N_{c}$ arguments

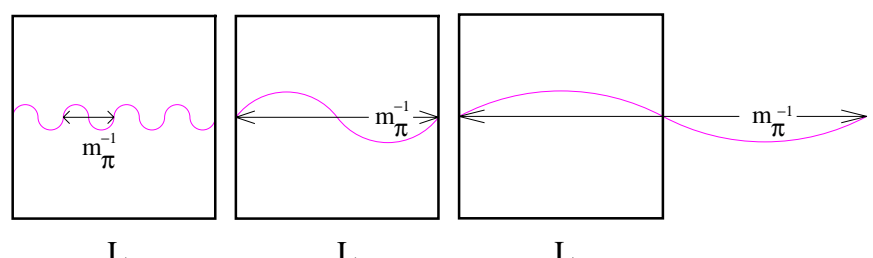

Fig. 21. Compton wavelength of the pions compared to the lattice size in the three regimes discussed in the text

center). In both cases, however, chiral perturbation theory can be done in the usual way, the only difference with the infinite volume calculation is that momenta of the pions are quantized.

Finally when the quark mass is so small that $m_{\pi} L \ll 1$ and $F^{2} m_{\pi}^{2} V<1$, then the standard chiral perturbation theory breaks down (Fig. 21 right). The zero-mode (i.e. the constant field configuration) of the pion field becomes non-perturbative signalling that spontaneous symmetry breaking does not occur in a finite volume. It was realized a long time ago by Gasser and Leutwyler 61] that the perturbative series can be reordered by factoring out the constant field configurations and treating them as collective variables:

$$
U=U_{0} U_{\xi}=U_{0} e^{i 2 \xi(x) / F} \quad \int d x \xi(x)=0 .
$$

The path integral then involves exact integrals over the flavour group manifold and a perturbative expansion for the non-zero modes:

$$
\mathcal{Z}=\int_{S U\left(N_{f}\right)} d U_{0} \int d \xi e^{-\mathcal{S}_{\chi}\left(U_{0}, \xi\right)}
$$

A convenient expansion for this regime is the so-called $\epsilon$-expansion where the power counting is:

$$
\frac{M_{P}}{4 \pi F} \sim\left(\frac{p}{4 \pi F}\right)^{2} \sim \frac{1}{4 \pi(L F)^{2}} \sim O\left(\epsilon^{2}\right) .
$$

The fact that powers of the pion mass and the momentum are counted differently implies a reordering of the chiral expansion:

$$
\tilde{\mathcal{L}}_{\chi}=\tilde{\mathcal{L}}_{\chi}^{(0)}+\tilde{\mathcal{L}}_{\chi}^{(2)}+\ldots
$$

While the leading order Lagrangian is the same as the leading one in (36), at next-to-leading order there are many differences. Out of the ten operators in the standard expansion only half appear in the $\epsilon$-regime at NLO:

$$
p \text {-regime } \quad \epsilon \text {-regime }
$$

$\begin{array}{lc}L_{1}\left\langle D_{\mu} U^{\dagger} D^{\mu} U\right\rangle^{2} & \sqrt{ } \\ L_{2}\left\langle D_{\mu} U^{\dagger} D_{\nu} U\right\rangle\left\langle D^{\mu} U^{\dagger} D^{\nu} U\right\rangle & \sqrt{ } \\ L_{3}\left\langle D_{\mu} U^{\dagger} D^{\mu} U D_{\nu} U^{\dagger} D^{\nu} U\right\rangle & \sqrt{ } \\ L_{4}\left\langle D_{\mu} U^{\dagger} D^{\mu} U\right\rangle\left\langle U^{\dagger} \chi+\chi^{\dagger} U\right\rangle & \times \\ L_{5}\left\langle D_{\mu} U^{\dagger} D^{\mu} U\left(U^{\dagger} \chi+\chi^{\dagger} U\right)\right\rangle & \times \\ L_{6}\left\langle U^{\dagger} \chi+\chi^{\dagger} U\right\rangle^{2} & \times \\ L_{7}\left\langle U^{\dagger} \chi-\chi^{\dagger} U\right\rangle^{2} & \times \\ L_{8}\left\langle\chi^{\dagger} U \chi^{\dagger} U+U^{\dagger} \chi U^{\dagger} \chi\right\rangle & \times \\ i L_{9}\left\langle F_{R}^{\mu \nu} D_{\mu} U D_{\nu} U^{\dagger}+F_{L}^{\mu \nu} D_{\mu} U^{\dagger} D_{\nu} U\right\rangle & \sqrt{ } \\ L_{10}\left\langle U^{\dagger} F_{R}^{\mu \nu} U F_{L \mu \nu}\right\rangle & \sqrt{ }\end{array}$

with $\chi \equiv M U \quad u_{\mu} \equiv i \partial_{\mu} U U^{\dagger}$. An even more dramatic simplification occurs in the weak Hamiltonian.

A number of quantities have been computed to NLO in the $\epsilon$-expansion both in the full and quenched theories: quark condensate, meson propagators in a $\theta$ vacuum and in fixed topology [62]. More recently three-point functions including the $\Delta S=1$ Hamiltonian have been obtained also at NLO 63].

The matching between lattice data and $\chi \mathrm{PT}$ in the $\epsilon$-regime (i.e. as close as possible to the chiral limit in a finite volume) should provide determinations of the lowenergy couplings with a minimal systematic uncertainty from the chiral extrapolation. Work in this direction is being pursued actively 64 .

\section{The beast: Unquenching}

Lattice QCD simulations in the quenched approximation have achieved a remarkable level of maturity and there are now many quantities:

- Light hadron spectrum

- Strange quark physics: $m_{s}, F_{K}$

- Charm quark physics: $m_{c}, F_{D_{s}}$, quarkonium levels

- Running coupling

$-\ldots$ 

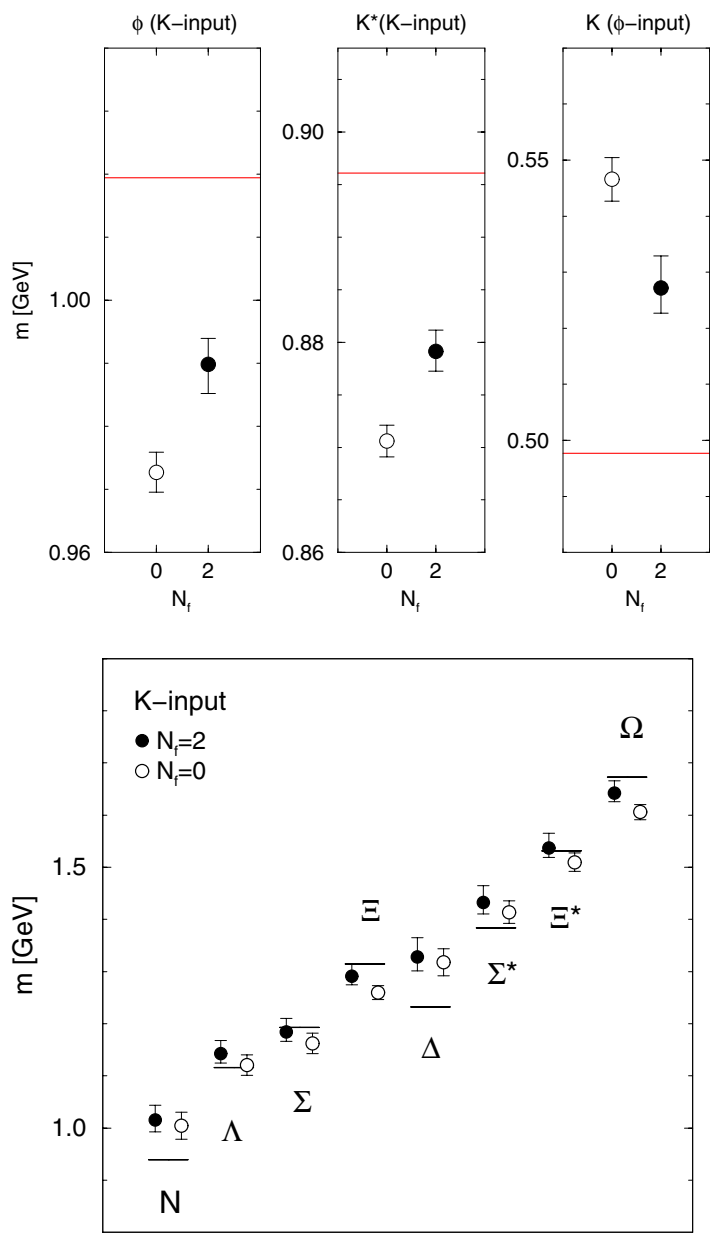

Fig. 22. Spectroscopy calculations in full QCD with $N_{f}=2$ and quenched QCD from 65.

that are known up to a few per cent statistical accuracy, with no other systematic uncertainty than that associated to the quenched approximation itself.

Several large collaborations with $O(1)$ Tflop computer power (CPPACS, JLQCD, MILC, UKQCD, ...) have produced in recent years many results beyond the quenched approximation with $N_{f}=2$ mostly. Figure 22 from 65 show some spectroscopy calculations with $N_{f}=2$. Although the results seem to come closer to experiment than the quenched results, the situation is far from satisfactory, due to the large systematic uncertainties. Most simulations have used rather coarse lattice spacings $(a \geq 0.09 \mathrm{fm})$ and are quite far from the chiral limit $\left(\frac{m_{\pi}}{m_{\rho}}=0.6-0.8\right)$.

Last year new simulations were reported with $N_{f}=3$ and an "improved" staggered action at $a=1 / 8 \mathrm{fm} 66$. Their main results are summarized in Fig. 23

Although these results are exciting, particularly because quark masses are significantly lighter than in previous simulations $\left(m_{\pi} / m_{\rho}=0.3 !\right)$, questions of principle remain concerning the action. For instance fundamental issues such as the locality of the staggered action are not fully understood, as mentioned in the introduction.

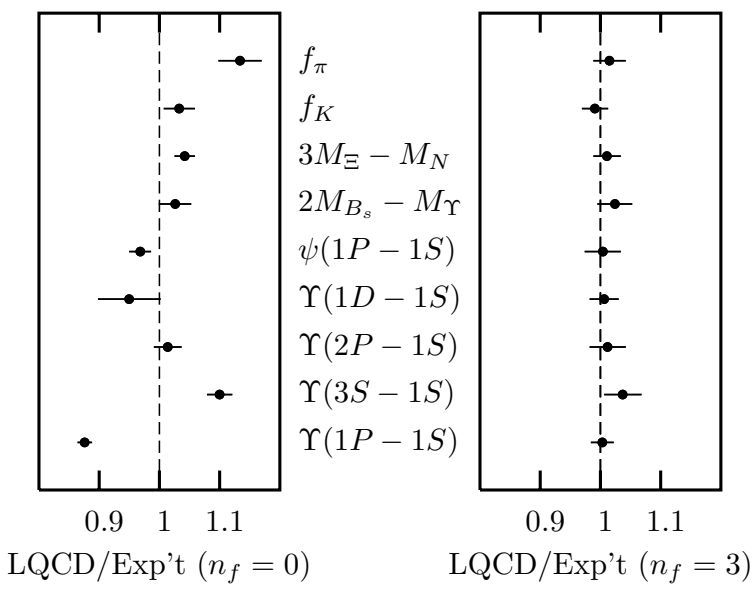

Fig. 23. Results from a $N_{f}=3$ simulation with "improved" staggered fermions at $a=0.125 \mathrm{fm}$. Taken from 66]

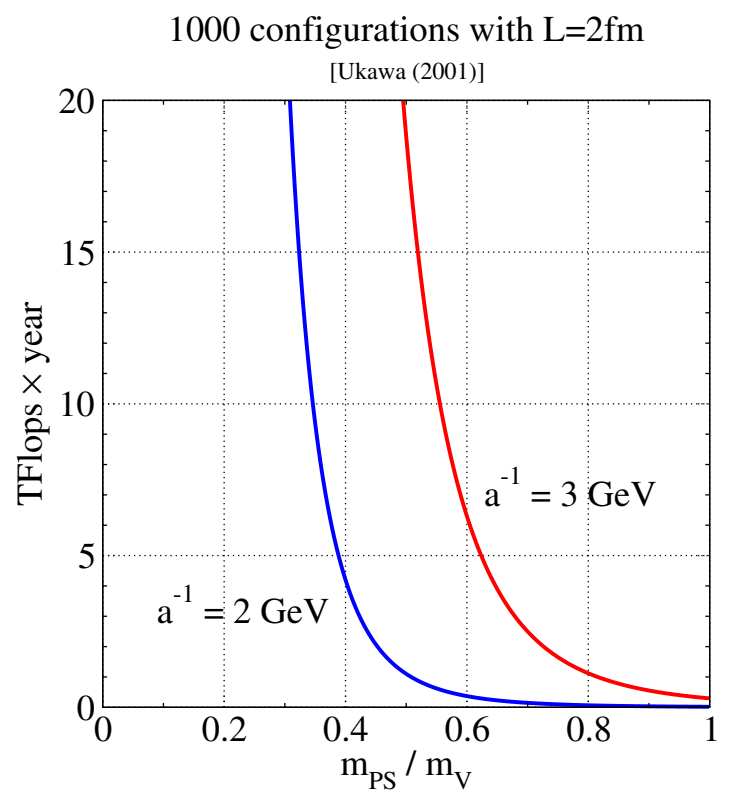

Fig. 24. Results from a $N_{f}=3$ simulation with "improved" staggered fermions at $a=0.125 \mathrm{fm}$. Taken from 66

On the other hand, the use of a perfectly safe fermion action such as the improved Wilson action faces enormous difficulties to reach such a chiral regime. This issue was the topic of a pannel discussion in the Berlin 2001 lattice conference. Figure 24 from 67] shows the expected computer power that will be needed to get 1000 thermalized unquenched configurations in a lattice of extent $L=2 \mathrm{fm}$ as a function of the ratio $m_{\pi} / m_{\rho}$. Although it was expected that algorithms should get more expensive with lighter quark masses, the increase in cost is quite spectacular. The empirical result for the scaling of the cost with the quark mass and the volume is, in Teraflop year,

$$
\frac{\text { \#oper. }}{\text { conf. }} \sim 3\left[\frac{140 \mathrm{MeV}}{m_{\pi}}\right]^{6}\left[\frac{L}{3 \mathrm{fm}}\right]^{5}\left[\frac{0.1 \mathrm{fm}}{a}\right]^{7} \mathrm{Tfy}
$$




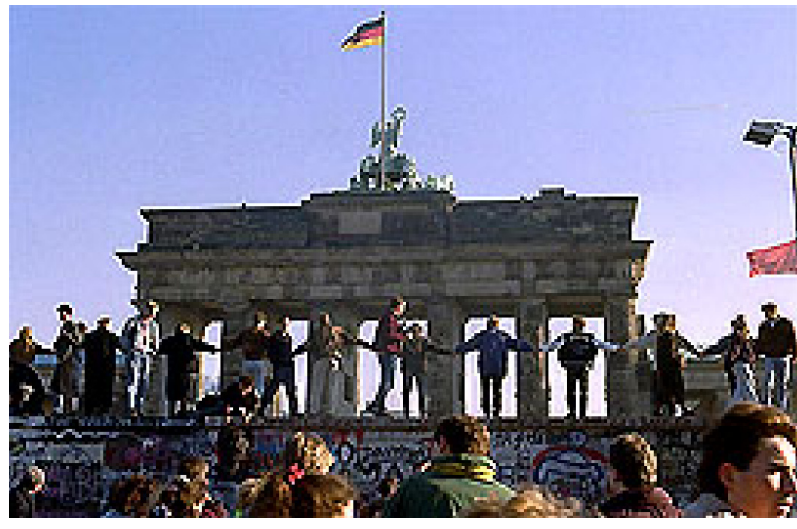

Fig. 25. Fall of the Berlin Wall

Next generation of computers will be in the range of $\mathcal{O}(10)$ Tflops (QCDOC, ApeNEXT). This will allow to reach $\frac{m_{\pi}}{m_{\rho}} \sim 0.4-0.6$ and $a^{-1}=2-3 \mathrm{GeV}$ in the next few years, which will probably be still too far from the physical point. Unless we do get smarter probably another order of magnitude in computer power will still be needed to arrive to the state-of-the-art quenched simulations of today with present algorithms. One should however keep in mind that there is a large potential gain by improvements in the algorithms and new ideas have been put forward in the past year 68,69$]$. Also there has been a proliferation on improved actions, which show a similar scaling as that in (43), but a better starting point [3. In many cases, however, questions of principle remain to be understood in these cases.

\section{Conclusions}

Lattice Field Theory is a mature and active field which has the best chance to answer non-perturbative questions in QCD and other gauge theories from first principles. Progress in recent years has been remarkable thanks to

- Exponential improvement in computer power

- Improvements in algorithms, actions and the questions being asked

Many quantities have been computed in the quenched approximation to a few per cent accuracy with full control over systematic errors: light hadron spectrum, $\Lambda, m_{s}, F_{K}$, $m_{c}, F_{D_{s}}$, etc A similar standard in unquenched simulations will take longer due to the Berlin wall of Fig. 24 but as in Fig. 25] we also hope to succeed!

Acknowledgements. I wish to thank P.H. Damgaard, L. Giusti, J. Heitger, K. Jansen, M. Laine, L. Lellouch, M. Lüscher, R. Sommer, C. Tarantino, P. Weisz and H. Wittig for many useful discussions on the topics covered in this review.

\section{References}

1. H.B. Nielsen and M. Ninomiya: Nucl. Phys. B 185, 20 (1981)
2. LATFOR initiative: http://www.zeuthen.desy.de/latfor

3. Proceedings of the International Conference Lattice 2003 (Tsukuba)

4. D.B. Kaplan, E. Katz, and M. Unsal: JHEP 0305 (2003), hep-lat/0302017; A.G. Cohen et al.: JHEP 0308 (2003) 024 and hep-lat/0307012

5. L. Giusti: parallel contribution to this conference, hep$\mathrm{ph} / 0312006$

6. J. Heitger: parallel contribution to this conference, hepph/0311045

7. S. Stone: plenary contribution to this conference, hep$\mathrm{ph} / 0310153$

8. C. Tarantino: parallel contribution to this conference, hepph/0310241

9. H. Wittig: parallel contribution to this conference, hepph/0310329

10. M. Lüscher, P. Weisz, and K. Symanzik: Nucl. Phys. B 173, 365 (1980); M. Lüscher: Nucl. Phys. B 180, 317 (1981)

11. M. Caselle et al: Nucl. Phys. B 486, 245 (1997)

12. M. Lüscher and P. Weisz: JHEP 0109, 010 (2001)

13. M. Lüscher and P. Weisz: JHEP 0207, 049 (2002)

14. S. Kratochvila and P. de Forcrand: Nucl. Phys. B 671, 103 (2003)

15. For recent reviews see M.G. Alford: Nucl. Phys. Proc. Suppl. 117, 65 (2003) and E. Laermann and O. Philipsen: hep-ph/0303042

16. Z. Fodor and S.D. Katz: Phys. Lett. B 534, 87 (2002)

17. C.R. Allton et al.: Phys. Rev. D 66, 074507 (2002)

18. P. de Forcrand and O. Philipsen: Nucl. Phys. B 642, 290 (2002) and Nucl. Phys. B 673, 170 (2003)

19. K. Symanzik: Nucl. Phys. B 226, 187 (1983)

20. See for example reviews M. Lüscher: hep-lat/9711205 and S. Sharpe: hep-lat/9811006

21. R. Frezzotti and G.C. Rossi: hep-lat/0306014

22. P.H. Ginsparg and K.G. Wilson: Phys. Rev. D 25, 2649 (1982)

23. P. Hasenfratz: Nucl. Phys. Proc. Suppl. 63, 53 (1998)

24. V. Rubakov and M. Shaposnikov: Phys. Lett. B 125, 136 (1983)

25. C.G. Callan and J.A. Harvey: Nucl. Phys. B 250, 427 (1985)

26. D.B. Kaplan: Phys. Lett. B 288, 342 (1992), [heplat/9206013]

27. Y. Shamir: Nucl. Phys. B 406, 90 (1993), [heplat/9303005]; V. Furman and Y. Shamir: Nucl. Phys. B 439, 54 (1995) [hep-lat/9405004]

28. R. Narayanan and H. Neuberger: Nucl. Phys. B 412, 574 (1994), [hep-lat/9307006]; Nucl. Phys. B 443, 305 (1995) [hep-th/9411108]

29. H. Neuberger: Phys. Lett. B 417, 141 (1998), [heplat/9707022]; ibid. 427, 353 (1998), [hep-lat/9801031]; Phys. Rev. D 57, 5417 (1998), [hep-lat/9710089]

30. P. Hernández, K. Jansen, and M. Lüscher: Nucl. Phys. B 552, 363 (1999)

31. M. Lüscher: Phys. Lett. B 428, 342 (1998), [heplat/9802011]

32. P. Hasenfratz, V. Laliena, and F. Niedermayer: Phys. Lett. B 427, 125 (1998)

33. P. Hasenfratz: Nucl. Phys. B 525, 401 (1998), [heplat/9802007]

34. R. Frezzotti et al.: JHEP 0108, 058 (2001); D. Becirevic et al.: Phys. Lett. B 487, 74 (2000); M. Guagnelli et al.: Nucl. Phys. Proc. Suppl. 106, 320 (2002) 
35. A. Aoki et al. [JLQCD Collaboration]: Phys. Rev. Lett. 80, 5271 (1998); G. Kilcup, R. Gupta, and S. Sharpe: Phys. Rev. D 57, 1654 (1998)

36. N. Garron et al.: hep-ph/0306295; T. Degrand, for MILC Coll.: hep-lat/0309026

37. E.V. Shuryak and J.M. Verbaarschot: Nucl. Phys. A 560, 306 (1993); J.J.M. Verbaarschot and I. Zahed: Phys. Rev. Lett. 70, 3852 (1993) and J.J.M. Verbaarschot: Phys. Rev. Lett. 72, 2531 (1994)

38. For reviews see J.J. Verbaarschot and T. Wettig: Anu. Rev. Nucl. Part. Sci. 50, 343 (2000) and P.H. Damgaard: Nucl. Phys. B (Proc. Suppl.) 106, 29 (2002)

39. W. Bietenholz, K. Jansen, and S. Shcheredin: JHEP 0307, 033 (2003)

40. L. Giusti et al.: JHEP 0311, 023 (2003)

41. J. Rolf and S. Sint [ALPHA Coll.]: JHEP 0212, 007 (2002)

42. A. Juttner and J. Rolf [ALPHA Coll.]: Phys. Lett. B 560, $59(2003)$

43. S. Choe et al. [QCD-TARO Coll.]: JHEP 0308, 022 (2003)

44. M. Guagnelli et al.: Phys. Lett. B 546, 237 (2002)

45. de Divitiis et al.: Nucl. Phys. B 675, 309 (2003) and Nucl. Phys. B 672, 372 (2003)

46. N. Isgur and M. Wise: Phys. Lett. B 232, 113 (1989); E. Eichten and B. Hill: Phys. Lett. B 234, 511 (1990); H. Georgi: Phys. Lett. B 240, 447 (1990)

47. L. Maiani, G. Martinelli, and C.T. Sachrajda: Nucl. Phys. B 368, 281 (1992)

48. R. Sommer: Nucl. Phys. Proc. Suppl. 119, 185 (2003)

49. J. Heitger, M. Kurth, and R. Sommer: Nucl. Phys. B 669, 173 (2003); J. Heitger and R. Sommer: hep-lat/0310035

50. J. Rolf et al.: hep-lat/0309072

51. T. Banks and A. Casher: Nucl. Phys. B 169, 103 (1980)

52. H. Leutwyler and A. Smilga: Phys. Rev. D 46, 5607 (1992)

53. S. Aoki et al. [JLQCD Coll.]: Phys. Rev. Lett. 91, 212001 (2003)

54. J. Gasser and H. Leutwyler: Nucl. Phys. B 250, 465 (1985)

55. See for instance the review A. Manohar: hep-ph/9802419

56. D.B. Kaplan and A. Manohar: Phys. Rev. Lett. 56, 2004 (1986); H. Leutwyler: Nucl. Phys. B 337, (1990)

57. J. Heitger, R. Sommer, and H. Wittig: Nucl. Phys. B 588, 377 (2000)
58. A.C. Irving et al. [UKQCD Coll.]: Phys. Lett. B 518, 243 (2001)

59. D.R. Nelson, G.T. Fleming, and G.W. Kilcup: Phys. Rev. Lett. 90, 021601 (2003)

60. F. Farchioni et al. [qq+q Coll.]: Eur. Phys. J. C 31, 227 (2003)

61. J. Gasser and H. Leutwyler: Phys. Lett. B 188, 477 (1987)

62. F.C. Hansen: Nucl. Phys. B 345, 685 (1990); F.C. Hansen and H. Leutwyler: Nucl. Phys. B 350, 201 (1991); J.C. Osborn, D. Toublan, and J.J. Verbaarschot: Nucl. Phys. B 540, 317 (1999), [hep-th/9806110]; P.H. Damgaard, J.C. Osborn, D. Toublan, and J.J. Verbaarschot: Nucl. Phys. B 547, 305 (1999), [hep-th/9811212]; P.H. Damgaard, M.C. Diamantini, P. Hernández and K. Jansen: Nucl. Phys. B 629, 445 (2002), [heplat/0112016]; P.H. Damgaard, P. Hernández, K. Jansen, M. Laine, and L. Lellouch: Nucl. Phys. B 656, 226 (2003), [hep-lat/0211020]

63. P. Hernández and M. Laine: JHEP 0301, (2003)

64. P.H. Damgaard et al.: Phys. Rev. D 61, 094503 (2000), [arXiv:hep-lat/9907016]; P. Hernandez, K. Jansen, and L. Lellouch: Phys. Lett. B 469, 198 (1999), [arXiv:heplat/9907022]; P. Hernandez et al.: JHEP 0107, 018 (2001), [arXiv:hep-lat/0106011]; T. DeGrand [MILC Collaboration]: Phys. Rev. D 64, 117501 (2001), [arXiv:heplat/0107014]; P. Hasenfratz et al.: Nucl. Phys. Proc. Suppl. 106, 751 (2002), [arXiv:hep-lat/0109007]; S. Prelovsek and K. Orginos [RBC Collaboration]: Nucl. Phys. Proc. Suppl. 119, 822 (2003), [arXiv:hep-lat/0209132]; L. Giusti et al.: Comput. Phys. Commun. 153, 31 (2003), [arXiv:heplat/0212012]; K.I. Nagai et al.: arXiv:hep-lat/0309051; T. Chiarappa et al.: arXiv:hep-lat/0309083; W. Bietenholz et al.: arXiv:hep-lat/0311012; L. Giusti et al.: heplat/0312012

65. S. Aoki et al. [JLQCD Collaboration]: Phys. Rev. D 68, 054502 (2003), [arXiv:hep-lat/0212039]

66. C.T.H. Davies et al. [HPQCD Collaboration]: arXiv:heplat/0304004

67. A. Ukawa: Nucl. Phys. B. (Proc. Suppl.) 106, 195 (2002)

68. M. Hasenbusch and K. Jansen: Nucl. Phys. B 659, 299 (2003) [arXiv:hep-lat/0211042]

69. M. Lüscher: JHEP 0305, 052 (2003), [arXiv:heplat/0304007] and arXiv:hep-lat/0310048 